\title{
On optimal solution error covariances in variational data assimilation problems
}

\author{
I.Yu. Gejadze ${ }^{\text {a }}$, F.-X. Le Dimet ${ }^{\text {b }}$, V. Shutyaev ${ }^{\text {c,* }}$

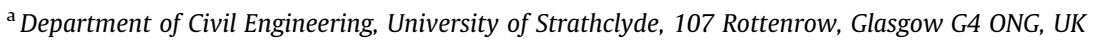 \\ ${ }^{\mathrm{b}}$ MOISE Project (CNRS, INRIA, UJF, INPG); LJK, Université Joseph Fourier, BP 51, 38051 Grenoble Cedex 9, France \\ ${ }^{\mathrm{c}}$ Institute of Numerical Mathematics, Russian Academy of Sciences, 119333 Gubkina 8, Moscow, Russia
}

\section{Introduction}

The methods of data assimilation (DA) have become an important tool for analysis of complex physical phenomena in various fields of science and technology. These methods allow us to combine mathematical models, data resulting from instrumental observations and a priori information. The problems of variational DA can be formulated as optimal control problems (e.g. $[10,12])$ to find unknown model parameters such as initial and/or boundary conditions, right-hand sides in the model equations (forcing terms), and distributed coefficients. A necessary optimality condition reduces an optimal control problem to an optimality system which includes inexact input functions; hence the error in the optimal solution. In this paper, assuming a perfect model, we consider two types of input errors: the background error and the observation error. It is an important theoretical and practical task to evaluate statistical properties of the optimal solution error. For example, its covariance can be used for estimating the efficiency of DA in terms of reducing uncertainty in model parameters and, therefore, in the model output.

The error in the optimal solution can be derived through the errors in the input data using the Hessian of an auxiliary DA problem [6,11]. If errors in the input data are random and subjected to the normal distribution, then for a linearized finitedimensional problem (tangent linear approximation of the discretized model) the covariance matrix of the analysis (optimal estimation of the initial condition) error is given by the inverse of the Hessian matrix of the cost functional (see e.g. $[7,9,17,21,22])$. In [6], a similar result was obtained for the continuous (both in time and space) operator formulation. We have shown that in the nonlinear case the analysis error covariance operator can be approximated by the inverse Hessian 
of the auxiliary DA problem based on the tangent linear model (TLM) constraints. We have also demonstrated that this approximation could be sufficiently accurate even though the tangent linear hypothesis is not valid.

This paper presents a generalization of the theoretical results reported in [6] to parameter estimation problems for a nonlinear evolution model. These problems are common inverse problems considered in geophysics $[20,25]$ and in engineering applications [1]. Here we derive the relationship between the optimal solution error covariance and the inverse Hessian of the auxiliary DA problem in a continuous operator form. The algorithm based on the quasi-Newton BFGS method (also reported in [6]) is adapted for constructing the optimal solution error covariance matrix for parameter estimation problems. This process is greatly accelerated by preconditioning the Hessian of the auxiliary DA problem, whereas the preconditioner is also defined in a general operator form.

For numerical analysis we use the one-dimensional (1D) nonlinear convection-diffusion model. The algorithm was applied to compute the covariance matrix for the diffusion coefficient and boundary flux estimation problems. The numerical results reveal interesting features of these problems in terms of identifiability, even for a simple evolution model. All numerical results have been verified using the fully nonlinear ensemble method [6]. Thus, we confirm that in the nonlinear case the optimal solution error covariance can be approximated by the inverse Hessian of the auxiliary DA problem ('H-covariance') beyond the validity of the tangent linear hypothesis.

The generalization of the theoretical results to the case of model errors is given in [19]. The relevant work discussing an estimate of posterior error fields in DA is given in [18].

This paper is organized as follows. In Section 2, we give the statement of the variational DA problem for a nonlinear evolution model to estimate the model parameters. In Section 3, the equation for the optimal solution error is derived through the errors of the input data. In Section 4 we derive the formulas for the optimal solution error covariance operator through the covariance operators of the input data errors using the Hessian of the auxiliary DA problem. A general case is considered in Section 4.1. Then, it is illustrated by the examples given for the 1D convection-diffusion model: the diffusion coefficient estimation problem is considered in Section 4.2 and the boundary flux estimation problem in Section 4.3. Details of numerical implementation are presented in Section 5 (for basic implementation details we also refer to [6]). We describe: in Section 5.1 a method for specifying the background error covariance matrix, in Section 5.2 - the preconditioning of the Hessian of the auxiliary DA problem and in Section 5.3 - other relevant implementation issues. Numerical analysis is presented in Section 6. In Section 6.1 we analyse the diffusion coefficient estimation problem, in Section 6.2 - the boundary flux estimation problem. In Section 6.3 we compare the convergence rates achieved with and without preconditioning for some numerical tests performed earlier. The main results are discussed in the Conclusions.

From this point on we shall refer to 'optimal solution error covariance/variance' simply as 'covariance/variance'.

\section{Statement of the problem}

Consider the mathematical model of a physical process that is described by the evolution problem

$$
\left\{\begin{array}{l}
\frac{\partial \varphi}{\partial t}=F(\varphi, \lambda)+f, \quad t \in(0, T), \\
\left.\varphi\right|_{t=0}=u,
\end{array}\right.
$$

where $\varphi=\varphi(t)$ is the unknown function belonging for any $t$ to a Hilbert space $X, u \in X, F$ is a nonlinear operator mapping $Y \times Y_{p}$ into $Y$ with $Y=L_{2}(0, T ; X),\|\cdot\|_{Y}=(\cdot, \cdot)_{Y}^{1 / 2}, Y_{p}$ is a Hilbert space (space of control parameters, or control space), $f \in Y$. Suppose that for given $u \in X, f \in Y$ and $\lambda \in Y_{p}$ there exists a unique solution $\varphi \in Y$ to (2.1). The function $\lambda$ is an unknown model parameter.

Let us introduce the functional

$$
S(\lambda)=\frac{1}{2}\left(V_{1}\left(\lambda-\lambda_{b}\right), \lambda-\lambda_{b}\right)_{Y_{p}}+\frac{1}{2}\left(V_{2}\left(C \varphi-\varphi_{o b s}\right), C \varphi-\varphi_{o b s}\right)_{Y_{o b s}}
$$

where $\lambda_{b} \in Y_{p}$ is a prior (background) function, $\varphi_{o b s} \in Y_{o b s}$ is a prescribed function (observational data), $Y_{o b s}$ is a Hilbert space (observation space), $C: Y \rightarrow Y_{o b s}$ is a linear bounded observation operator, $V_{1}: Y_{p} \rightarrow Y_{p}$ and $V_{2}: Y_{o b s} \rightarrow Y_{o b s}$ are symmetric positive definite bounded operators.

Let us consider the following DA problem with the aim to estimate the parameter $\lambda$ : for given $u \in X, f \in Y$, find $\lambda \in Y_{p}$ and $\varphi \in Y$ such that they satisfy (2.1), and on the set of solutions to (2.1), the functional $S(\lambda)$ takes the minimum value, i.e.

$$
\left\{\begin{array}{l}
\frac{\partial \varphi}{\partial t}=F(\varphi, \lambda)+f, \quad t \in(0, T), \\
\left.\varphi\right|_{t=0}=u \\
S(\lambda)=\inf _{v \in Y_{p}} S(v) .
\end{array}\right.
$$

We suppose that the solution of (2.3) exists. Let us note that the solvability of the parameter estimation problems (or identifiability) has been addressed, e.g., in [2,14]. To derive the optimality system, we assume the solution $\varphi$ and the operator $F(\varphi, \lambda)$ in (2.1) and (2.2) are regular enough, and for $v \in Y_{p}$ find the gradient of the functional $S$ with respect to $\lambda$ :

$$
S^{\prime}(\lambda) v=\left(V_{1}\left(\lambda-\lambda_{b}\right), v\right)_{Y_{p}}+\left(V_{2}\left(C \varphi-\varphi_{o b s}\right), C \phi\right)_{Y_{o b s}}=\left(V_{1}\left(\lambda-\lambda_{b}\right), v\right)_{Y_{p}}+\left(C^{*} V_{2}\left(C \varphi-\varphi_{o b s}\right), \phi\right)_{Y}
$$


where $\phi$ is the solution to the problem:

$$
\left\{\begin{array}{l}
\frac{\partial \phi}{\partial t}=F_{\varphi}^{\prime}(\varphi, \lambda) \phi+F_{\lambda}^{\prime}(\varphi, \lambda) v, \quad t \in(0, T), \\
\left.\phi\right|_{t=0}=0
\end{array}\right.
$$

Here $F_{\varphi}^{\prime}(\varphi, \lambda): Y \rightarrow Y, F_{\lambda}^{\prime}(\varphi, \lambda): Y_{p} \rightarrow Y$ are the Frechet derivatives of $F$ with respect to $\varphi$ and $\lambda$, correspondingly, and $C^{*}$ is the adjoint operator to $C$ defined by $(C \varphi, \psi)_{Y_{o b s}}=\left(\varphi, C^{*} \psi\right)_{Y}, \varphi \in Y, \psi \in Y_{o b s}$.

Let us consider the adjoint operator $\left(F_{\varphi}^{\prime}(\varphi, \lambda)\right)^{*}: Y \rightarrow Y$ and introduce the adjoint problem:

$$
\left\{\begin{array}{l}
-\frac{\partial \varphi^{*}}{\partial t}-\left(F_{\varphi}^{\prime}(\varphi, \lambda)\right)^{*} \varphi^{*}=-C^{*} V_{2}\left(C \varphi-\varphi_{o b s}\right), \quad t \in(0, T), \\
\left.\varphi^{*}\right|_{t=T}=0 .
\end{array}\right.
$$

Then (2.4) with (2.5) and (2.6) gives

$$
S^{\prime}(\lambda) v=\left(V_{1}\left(\lambda-\lambda_{b}\right), v\right)_{Y_{p}}-\left(\varphi^{*}, F_{\lambda}^{\prime}(\varphi, \lambda) v\right)_{Y}=\left(V_{1}\left(\lambda-\lambda_{b}\right), v\right)_{Y_{p}}-\left(\left(F_{\lambda}^{\prime}(\varphi, \lambda)\right)^{*} \varphi^{*}, v\right)_{Y_{p}},
$$

where $\left(F_{\lambda}^{\prime}(\varphi, \lambda)\right)^{*}: Y \rightarrow Y_{p}$ is the adjoint operator to $F_{\lambda}^{\prime}(\varphi, \lambda)$. Therefore, the gradient of $S$ is defined by

$$
S^{\prime}(\lambda)=V_{1}\left(\lambda-\lambda_{b}\right)-\left(F_{\lambda}^{\prime}(\varphi, \lambda)\right)^{*} \varphi^{*}
$$

From (2.4)-(2.6) and (2.7) we get the optimality system (the necessary optimality conditions):

$$
\begin{aligned}
& \left\{\begin{array}{l}
\frac{\partial \varphi}{\partial t}=F(\varphi, \lambda)+f, \quad t \in(0, T), \\
\left.\varphi\right|_{t=0}=u
\end{array}\right. \\
& \left\{\begin{array}{l}
-\frac{\partial \varphi^{*}}{\partial t}-\left(F_{\varphi}^{\prime}(\varphi, \lambda)\right)^{*} \varphi^{*}=-C^{*} V_{2}\left(C \varphi-\varphi_{o b s}\right), \quad t \in(0, T), \\
\left.\varphi^{*}\right|_{t=T}=0
\end{array}\right. \\
& V_{1}\left(\lambda-\lambda_{b}\right)-\left(F_{\lambda}^{\prime}(\varphi, \lambda)\right)^{*} \varphi^{*}=0
\end{aligned}
$$

We assume that the system (2.8)-(2.10) has a unique solution. Suppose that $\lambda_{b}=\bar{\lambda}+\xi_{1}, \varphi_{o b s}=C \bar{\varphi}+\xi_{2}$, where $\xi_{1} \in Y_{p}, \xi_{2} \in Y_{o b s}$, and $\bar{\varphi}$ is the ("true") solution to the problem (2.1) with $\lambda=\bar{\lambda}$ :

$$
\left\{\begin{array}{l}
\frac{\partial \bar{\varphi}}{\partial t}=F(\bar{\varphi}, \bar{\lambda})+f, \quad t \in(0, T) \\
\left.\bar{\varphi}\right|_{t=0}=u
\end{array}\right.
$$

The functions $\xi_{1}, \xi_{2}$ represent the errors of the input data $\lambda_{b}$ and $\varphi_{\text {obs }}$ (background and observation error, respectively).

If the observation operator $C$ is nonlinear, i.e. $C \varphi=C(\varphi)$, then the right-hand-side of the adjoint Eq. (2.9) contains $\left(C_{\varphi}^{\prime}\right)^{*}$ instead of $C^{* *}$ and all the analysis presented below is similar.

\section{Equation for the optimal solution error}

Let us derive the equation for the optimal solution error through the input data errors. Let $\delta \varphi=\varphi-\bar{\varphi}, \delta \lambda=\lambda-\bar{\lambda}$. Let us suppose that $F$ is continuously Frechet differentiable, and then there exist $\tilde{\varphi}=\bar{\varphi}+\tau(\varphi-\bar{\varphi}), \tilde{\lambda}=\bar{\lambda}+\tau(\lambda-\bar{\lambda}), \tau \in[0,1]$, such that the Taylor-Lagrange formula [13] is valid: $F(\varphi, \lambda)-F(\bar{\varphi}, \bar{\lambda})=F_{\varphi}^{\prime}(\tilde{\varphi}, \tilde{\lambda}) \delta \varphi+F_{\lambda}^{\prime}(\tilde{\varphi}, \tilde{\lambda}) \delta \lambda$. Then, from (2.11) and the optimality system (2.8)-(2.10), we obtain

$$
\begin{aligned}
& \left\{\begin{array}{l}
\frac{\partial \delta \varphi}{\partial t}-F_{\varphi}^{\prime}(\tilde{\varphi}, \tilde{\lambda}) \delta \varphi=F_{\lambda}^{\prime}(\tilde{\varphi}, \tilde{\lambda}) \delta \lambda, \quad t \in(0, T), \\
\left.\delta \varphi\right|_{t=0}=0,
\end{array}\right. \\
& \left\{\begin{array}{l}
-\frac{\partial \varphi^{*}}{\partial t}-\left(F_{\varphi}^{\prime}(\varphi, \lambda)\right)^{*} \varphi^{*}=-C^{*} V_{2}\left(C \delta \varphi-\xi_{2}\right), \quad t \in(0, T), \\
\left.\varphi^{*}\right|_{t=T}=0,
\end{array}\right. \\
& V_{1}\left(\delta \lambda-\xi_{1}\right)-\left(F_{\lambda}^{\prime}(\varphi, \lambda)\right)^{*} \varphi^{*}=0 .
\end{aligned}
$$

Note that $\tilde{\varphi}=\bar{\varphi}+\tau \delta \varphi, \varphi=\bar{\varphi}+\delta \varphi, \tilde{\lambda}=\bar{\lambda}+\tau \delta \lambda, \lambda=\bar{\lambda}+\delta \lambda$. The system (3.1)-(3.3) may be written in the form:

$$
\begin{aligned}
& \left\{\begin{array}{l}
\frac{\partial \delta \varphi}{\partial t}-F_{\varphi}^{\prime}(\bar{\varphi}, \bar{\lambda}) \delta \varphi=F_{\lambda}^{\prime}(\bar{\varphi}, \bar{\lambda}) \delta \lambda+\xi_{3}, \quad t \in(0, T), \\
\left.\delta \varphi\right|_{t=0}=0,
\end{array}\right. \\
& \left\{\begin{array}{l}
-\frac{\partial \varphi^{*}}{\partial t}-\left(F_{\varphi}^{\prime}(\bar{\varphi}, \bar{\lambda})\right)^{*} \varphi^{*}=-C^{*} V_{2}\left(C \delta \varphi-\xi_{2}\right)+\xi_{4}, \quad t \in(0, T), \\
\left.\varphi^{*}\right|_{t=T}=0,
\end{array}\right. \\
& V_{1}\left(\delta \lambda-\xi_{1}\right)-\left(F_{\lambda}^{\prime}(\bar{\varphi}, \bar{\lambda})\right)^{*} \varphi^{*}=\xi_{5},
\end{aligned}
$$


where

$$
\begin{aligned}
& \xi_{3}=\left[F_{\varphi}^{\prime}(\tilde{\varphi}, \tilde{\lambda})-F_{\varphi}^{\prime}(\bar{\varphi}, \bar{\lambda})\right] \delta \varphi+\left[F_{\lambda}^{\prime}(\tilde{\varphi}, \tilde{\lambda})-F_{\lambda}^{\prime}(\bar{\varphi}, \bar{\lambda})\right] \delta \lambda, \\
& \xi_{4}=\left[\left(F_{\varphi}^{\prime}(\varphi, \lambda)\right)^{*}-\left(F_{\varphi}^{\prime}(\bar{\varphi}, \bar{\lambda})\right)^{*}\right] \varphi^{*}, \quad \xi_{5}=\left[\left(F_{\lambda}^{\prime}(\varphi, \lambda)\right)^{*}-\left(F_{\lambda}^{\prime}(\bar{\varphi}, \bar{\lambda})\right)^{*}\right] \varphi^{*} .
\end{aligned}
$$

For fixed $\xi_{i}(i=1,2,3,4,5)$, excluding $\delta \varphi$ and $\varphi^{*}$ from (3.4)-(3.6), we derive a single equation for $\delta \lambda$ (see (3.17) below). Let us introduce the operator $H: Y_{p} \rightarrow Y_{p}$ defined by the successive solutions of the following problems:

$$
\begin{aligned}
& \left\{\begin{array}{l}
\frac{\partial \psi}{\partial t}-F_{\varphi}^{\prime}(\bar{\varphi}, \bar{\lambda}) \psi=F_{\lambda}^{\prime}(\bar{\varphi}, \bar{\lambda}) v, \quad t \in(0, T), \\
\left.\psi\right|_{t=0}=0,
\end{array}\right. \\
& \left\{\begin{array}{l}
-\frac{\partial \psi^{*}}{\partial t}-\left(F_{\varphi}^{\prime}(\bar{\varphi}, \bar{\lambda})\right)^{*} \psi^{*}=-C^{*} V_{2} C \psi, \quad t \in(0, T), \\
\left.\psi^{*}\right|_{t=T}=0,
\end{array}\right. \\
& H v=V_{1} v-\left(F_{\lambda}^{\prime}(\bar{\varphi}, \bar{\lambda})\right)^{*} \psi^{*} .
\end{aligned}
$$

We show next that $H$ is the Hessian of an auxiliary data assimilation problem based on the tangent linear model constraints. Below we introduce four auxiliary operators $R_{1}, R_{2}, R_{3}, R_{4}$. Let $R_{1}=V_{1}$. Let us introduce the operator $R_{2}: Y_{\text {obs }} \rightarrow Y_{p}$ acting on the functions $g \in Y_{o b s}$ according to the formula

$$
R_{2} g=\left(F_{\lambda}^{\prime}(\bar{\varphi}, \bar{\lambda})\right)^{*} \theta^{*},
$$

where $\theta^{*}$ is the solution to the adjoint problem

$$
\left\{\begin{array}{l}
-\frac{\partial \theta^{*}}{\partial t}-\left(F_{\varphi}^{\prime}(\bar{\varphi}, \bar{\lambda})\right)^{*} \theta^{*}=C^{*} V_{2} g, \quad t \in(0, T), \\
\left.\theta^{*}\right|_{t=T}=0 .
\end{array}\right.
$$

The operator $R_{3}: Y \rightarrow Y_{p}$ is defined on the functions $q \in Y$ as follows:

$$
\begin{aligned}
& \left\{\begin{array}{l}
\frac{\partial \theta_{1}}{\partial t}-F_{\varphi}^{\prime}(\bar{\varphi}, \bar{\lambda}) \theta_{1}=q, \quad t \in(0, T), \\
\left.\theta_{1}\right|_{t=0}=0,
\end{array}\right. \\
& \left\{\begin{array}{l}
-\frac{\partial \theta_{1}^{*}}{\partial t}-\left(F_{\varphi}^{\prime}(\bar{\varphi}, \bar{\lambda})\right)^{*} \theta_{1}^{*}=-C^{*} V_{2} C \theta_{1}, \quad t \in(0, T), \\
\left.\theta_{1}^{*}\right|_{t=T}=0,
\end{array}\right. \\
& R_{3} q=\left(F_{\lambda}^{\prime}(\bar{\varphi}, \bar{\lambda})\right)^{*} \theta_{1}^{*} .
\end{aligned}
$$

The operator $R_{4}: Y \rightarrow Y_{p}$ is defined on the functions $q \in Y$ as

$$
\begin{aligned}
& \begin{cases}-\frac{\partial \theta_{2}^{*}}{\partial t}-\left(F_{\varphi}^{\prime}(\bar{\varphi}, \bar{\lambda})\right)^{*} \theta_{2}^{*} & =q, \quad t \in(0, T), \\
\left.\theta_{2}^{*}\right|_{t=T} & =0,\end{cases} \\
& R_{4} q=\left(F_{\lambda}^{\prime}(\bar{\varphi}, \bar{\lambda})\right)^{*} \theta_{2}^{*} .
\end{aligned}
$$

From (3.7)-(3.16) we conclude that the system (3.4)-(3.6) is equivalent to the single equation for $\delta \lambda$ :

$$
H \delta \lambda=R_{1} \xi_{1}+R_{2} \xi_{2}+R_{3} \xi_{3}+R_{4} \xi_{4}+\xi_{5} .
$$

Each operator $R_{i}$ defines the contribution of the corresponding error $\xi_{i}$ into the right-hand-side of the error Eq. (3.17). This is the exact equation for $\delta \lambda$. Under the hypothesis that $H$ is invertible, we get

$$
\delta \lambda=T_{1} \xi_{1}+T_{2} \xi_{2}+T_{3} \xi_{3}+T_{4} \xi_{4}+T_{5} \xi_{5},
$$

where $T_{i}=H^{-1} R_{i}, i=1,2,3,4, T_{5}=H^{-1}, T_{1}: Y_{p} \rightarrow Y_{p}, T_{2}: Y_{o b s} \rightarrow Y_{p}, T_{3}, T_{4}: Y \rightarrow Y_{p}$.

The operators $T_{i}(i=1,2,3,4,5)$ are bounded, because the operators $R_{i}$ and the inverse Hessian $H^{-1}$ are supposed to be bounded. Each operator $T_{i}$ can be regarded as an error transfer operator which relates the corresponding error $\xi_{i}$ to the optimal solution error $\delta \lambda$.

Let us note that the functions $\varphi, \lambda, \tilde{\varphi}, \tilde{\lambda}$ in $3.1,3.2$ and 3.3 depend on $\xi_{1}, \xi_{2}$, so as a result, the terms $T_{3} \xi_{3}, T_{4} \xi_{4}, T_{5} \xi_{5}$ also depend nonlinearly on $\xi_{1}, \xi_{2}$, and it is not possible to represent $\delta \lambda$ through $\xi_{1}, \xi_{2}$ in an explicit form. To derive from (3.18) the covariance operator of $\delta \lambda$, we need to introduce some approximation of (3.18). Since $\tilde{\varphi}=\bar{\varphi}+\tau \delta \varphi, \varphi=\bar{\varphi}+\delta \varphi, \tilde{\lambda}=\bar{\lambda}+$ $\tau \delta \lambda, \lambda=\bar{\lambda}+\delta \lambda$, we assume that

$$
T_{3} \xi_{3} \approx 0, \quad T_{4} \xi_{4} \approx 0, \quad T_{5} \xi_{5} \approx 0,
$$

then (3.18) reduces to

$$
\delta \lambda \approx T_{1} \xi_{1}+T_{2} \xi_{2},
$$

which is equivalent to the system: 


$$
\begin{aligned}
& \left\{\begin{array}{l}
\frac{\partial \delta \varphi}{\partial t}-F_{\varphi}^{\prime}(\bar{\varphi}, \bar{\lambda}) \delta \varphi=F_{\lambda}^{\prime}(\bar{\varphi}, \bar{\lambda}) \delta \lambda, \quad t \in(0, T), \\
\left.\delta \varphi\right|_{t=0}=0,
\end{array}\right. \\
& \left\{\begin{array}{l}
-\frac{\partial \varphi^{*}}{\partial t}-\left(F_{\varphi}^{\prime}(\bar{\varphi}, \bar{\lambda})\right)^{*} \varphi^{*}=-C^{*} V_{2}\left(C \delta \varphi-\xi_{2}\right), \quad t \in(0, T), \\
\left.\varphi^{*}\right|_{t=T}=0,
\end{array}\right. \\
& V_{1}\left(\delta \lambda-\xi_{1}\right)-\left(F_{\lambda}^{\prime}(\bar{\varphi}, \bar{\lambda})\right)^{*} \varphi^{*}=0 .
\end{aligned}
$$

Taking into account the definition of $\xi_{3}, \xi_{4}, \xi_{5}$, it can be seen that the assumption (3.19) is equivalent to the first-order approximation of the Taylor-Lagrange formula under the hypothesis that $F$ is twice continuously Frechet differentiable [13]. Using this formula, the errors $\xi_{3}, \xi_{4}$ and $\xi_{5}$, may be expressed through the second derivatives of $F$, and the values of the norms of $T_{3} \xi_{3}, T_{4} \xi_{4}, T_{5} \xi_{5}$ can be estimated, thus giving the possibility that the linearization error can be assessed.

For fixed $\bar{\lambda}, \bar{\varphi}$, the problem (3.21)-(3.23) is the necessary optimality condition to the following DA problem: find $\delta \lambda$ and $\delta \varphi$ such that

$$
\left\{\begin{array}{l}
\frac{\partial \delta \varphi}{\partial t}-F_{\varphi}^{\prime}(\bar{\varphi}, \bar{\lambda}) \delta \varphi=F_{\lambda}^{\prime}(\bar{\varphi}, \bar{\lambda}) \delta \lambda, \quad t \in(0, T), \\
\left.\delta \varphi\right|_{t=0}=0 \\
S_{1}(\delta \lambda)=\inf _{v \in Y_{p}} S_{1}(v),
\end{array}\right.
$$

where

$$
S_{1}(\delta \lambda)=\frac{1}{2}\left(V_{1}\left(\delta \lambda-\xi_{1}\right), \delta \lambda-\xi_{1}\right)_{Y_{p}}+\frac{1}{2}\left(V_{2}\left(C \delta \varphi-\xi_{2}\right), C \delta \varphi-\xi_{2}\right)_{Y_{o b s}} .
$$

The Hessian $H$ of the functional (3.25) is defined on $v \in Y_{p}$ by (3.7)-(3.9). Note that for $\xi_{2}=0$ the operator $H$ coincides with the Hessian $\mathscr{H}$ of the original nonlinear DA problem on the exact solution $\bar{\lambda}$. The Hessian $H$ acts in $Y_{p}$ as a self-adjoint operator with domain of definition $D(H)=Y_{p}$. Moreover, because of the properties of $V_{1}, V_{2}$, the operator $H$ is always positive definite, and hence invertible.

The derivation here follows the reasoning of [6], where the initialization problem was considered. However, here the optimality system and, subsequently, definition of the Hessian $H$ (via differential problems) are different from [6]. In particular, they involve the adjoints to the Frechet derivatives of $F$ both with respect to the solution $\varphi$ and the parameter $\lambda$.

\section{Covariance operator as the inverse Hessian}

\subsection{General case}

Consider the error Eq. (3.20), where $T_{i}=H^{-1} R_{i}, i=1,2, T_{1}: Y_{p} \rightarrow Y_{p}, T_{2}: Y_{o b s} \rightarrow Y_{p}$. Below we suppose that the errors $\xi_{1}, \xi_{2}$ are normally distributed, unbiased, and mutually uncorrelated. Let us denote by $V_{\delta \lambda}$ the covariance operator $V_{\delta \lambda}=E\left[(\cdot, \delta \lambda)_{Y_{p}} \delta \lambda\right]$, and by $V_{\xi_{i}}$ the covariance operator of the corresponding error $\xi_{i}, i=1,2$, i.e. $V_{\xi_{1}} \cdot=E\left[\left(\cdot, \xi_{1}\right)_{Y_{p}} \xi_{1}\right]$, $V_{\xi_{2}} \cdot=E\left[\left(\cdot, \xi_{2}\right)_{Y_{o b s}} \xi_{2}\right]$, where $E$ is the expectation. For $V_{1}$ and $V_{2}$ in (2.2), we take $V_{1}=V_{\xi_{1}}^{-1}, V_{2}=V_{\xi_{2}}^{-1}$. From (3.20) we get

$$
V_{\delta \lambda} \approx V:=T_{1} V_{\xi_{1}} T_{1}^{*}+T_{2} V_{\xi_{2}} T_{2}^{*}
$$

To find the operator $V$, we need to construct the operators $T_{i} V_{\xi_{i}} T_{i}^{*}, i=1,2$.

Consider the operator $T_{1} V_{\xi_{1}} T_{1}^{*}$. Since $T_{1}=H^{-1} R_{1}$, we have $T_{1} V_{\xi_{1}} T_{1}^{*}=H^{-1} R_{1} V_{\xi_{1}} R_{1} H^{-1}$. Moreover, if $V_{1}=V_{\xi_{1}}^{-1}$, then

$$
T_{1} V_{\xi_{1}} T_{1}^{*}=H^{-1} R_{1} H^{-1} \text {. }
$$

Consider the operator $T_{2} V_{\xi_{2}} T_{2}^{*}$. Since $T_{2}=H^{-1} R_{2}$, then

$$
T_{2} V_{\xi_{2}} T_{2}^{*}=H^{-1} R_{2} V_{\xi_{2}} R_{2}^{*} H^{-1} .
$$

To determine $R_{2}^{*}$, consider the inner product $\left(R_{2} g, p\right)_{Y_{p}}, g \in Y_{o b s}, p \in Y_{p}$. From (3.10) and (3.11),

$$
\left(R_{2} g, p\right)_{Y_{p}}=\left(\left(F_{\lambda}^{\prime}(\bar{\varphi}, \bar{\lambda})\right)^{*} \theta^{*}, p\right)_{Y_{p}}=\left(C^{*} V_{2} g, \phi\right)_{Y}=\left(g, R_{2}^{*} p\right)_{Y_{o b s}},
$$

where $R_{2}^{*} p=V_{2} C \phi$, and $\phi$ is the solution to the problem

$$
\left\{\begin{array}{l}
\frac{\partial \phi}{\partial t}-F_{\varphi}^{\prime}(\bar{\varphi}, \bar{\lambda}) \phi=F_{\lambda}^{\prime}(\bar{\varphi}, \bar{\lambda}) p, \quad t \in(0, T), \\
\left.\phi\right|_{t=0}=0
\end{array}\right.
$$


Thus, the operator $T_{2} V_{\xi_{2}} T_{2}^{*}$ is defined by successive solutions of the following problems (for a given $v \in Y_{p}$ ):

$$
\begin{aligned}
& H p=v, \\
& \left\{\begin{array}{l}
\frac{\partial \phi}{\partial t}-F_{\varphi}^{\prime}(\bar{\varphi}, \bar{\lambda}) \phi=F_{\lambda}^{\prime}(\bar{\varphi}, \bar{\lambda}) p, \quad t \in(0, T), \\
\left.\phi\right|_{t=0}=0,
\end{array}\right. \\
& \left\{\begin{array}{l}
-\frac{\partial \theta^{*}}{\partial t}-\left(F_{\varphi}^{\prime}(\bar{\varphi}, \bar{\lambda})\right)^{*} \theta^{*}=C^{*} V_{2} V_{\xi_{2}} V_{2} C \phi, \quad t \in(0, T) \\
\left.\theta^{*}\right|_{t=T}=0,
\end{array}\right. \\
& H w=\left(F_{\lambda}^{\prime}(\bar{\varphi}, \bar{\lambda})\right)^{*} \theta^{*},
\end{aligned}
$$

then

$$
T_{2} V_{\xi_{2}} T_{2}^{*} v=w
$$

If $V_{2}=V_{\xi_{2}}^{-1}$, then $C^{*} V_{2} V_{\xi_{2}} V_{2} C=C^{*} V_{2} C$ and from (4.6) and (4.7) we obtain that

$$
\left(F_{\lambda}^{\prime}(\bar{\varphi}, \bar{\lambda})\right)^{*} \theta^{*}=H p-R_{1} p,
$$

where $H$ is the Hessian defined by 3.7, 3.8 and 3.9. From the definition of $R_{2}$, we then get

$$
R_{2} V_{\xi_{2}} R_{2}^{*}=H-R_{1}
$$

and

$$
T_{2} V_{\xi_{2}} T_{2}^{*}=H^{-1} R_{2} V_{\xi_{2}} R_{2}^{*} H^{-1}=H^{-1}\left(H-R_{1}\right) H^{-1} .
$$

From (4.2) and (4.9) the result for $V$ follows:

$$
V=T_{1} V_{\xi_{1}} T_{1}^{*}+T_{2} V_{\xi_{2}} T_{2}^{*}=H^{-1} H H^{-1}=H^{-1},
$$

i.e. the covariance operator $V_{\delta \lambda}$ is approximately the inverse Hessian. By this reason we refer to $V$ as the $H$-covariance.

Therefore, for the parameter estimation problem we obtain the same result as for the initialization (initial-value control) problem. It means that the numerical algorithm for computing the covariance matrix presented in [6] can be used in the case under consideration. Below the theory developed is illustrated by the examples given for the 1D convection-diffusion model.

\subsection{Diffusion coefficient estimation}

Let us consider the following evolution model:

$$
\left\{\begin{array}{l}
\frac{\partial \varphi}{\partial t}=F(\varphi, k)+f, \quad t \in(0, T), \quad x \in(0,1), \\
\left.\varphi\right|_{t=0}=u, \\
-\left.k \frac{\partial \varphi}{\partial x}\right|_{x=0}=0,\left.\quad k \frac{\partial \varphi}{\partial x}\right|_{x=1}=0,
\end{array}\right.
$$

where $F(\varphi, k)$ is the $1 \mathrm{D}$ convection-diffusion operator as follows:

$$
F(\varphi, k)=-\frac{\partial(w \varphi)}{\partial x}+\frac{\partial}{\partial x}\left(k \frac{\partial \varphi}{\partial x}\right)
$$

Above, $k=k(x)$ is the unknown diffusion coefficient, $u=u(x), w=w(t, x)$ and $f=f(t, x)$ are prescribed functions. Consider the functional $S(\lambda)$ defined by (2.2) with $Y_{p}=X=L_{2}(0,1)$, where $\lambda=k$. The DA problem is as follows: find the functions $\lambda=\lambda(x)$ and $\varphi=\varphi(t, x)$ such that they satisfy (4.11), and on the set of solutions to (4.11), the functional $S(\lambda)$ takes the minimum value. The space $Y_{o b s}$ and the corresponding observation term are the same as in (2.2). Note that $Y_{o b s}$ can be the whole space $Y=L_{2}(0, T ; X)$, or its subspace, and depends on the choice of the observation operator $C$ (related to the observation scheme). The details of specific observation schemes used in numerical experiments are discussed in Section 6.

The DA problem stated above has the same form as (2.3), therefore all results presented in Section 4.1 are directly applicable. Let us also notice that even though the evolution model (4.11) is linear in $\varphi$ ( $k$ does not depend on $\varphi$ ), the DA problem is nonlinear, because the operator $F(\varphi, \lambda)$ is nonlinear.

The gradient of the functional $S$ is defined by (2.4), where $\phi$ is the solution to (2.5) satisfying the homogeneous boundary conditions:

$$
\left.\frac{\partial \phi}{\partial x}\right|_{x=0}=\left.\frac{\partial \phi}{\partial x}\right|_{x=1}=0
$$

and the operators $F_{\varphi}^{\prime}(\varphi, \lambda), F_{\lambda}^{\prime}(\varphi, \lambda)$ are defined by

$$
F_{\varphi}^{\prime}(\varphi, \lambda) \phi=-\frac{\partial(w \phi)}{\partial x}+\frac{\partial}{\partial x}\left(\lambda \frac{\partial \phi}{\partial x}\right), \quad F_{\lambda}^{\prime}(\varphi, \lambda) v=\frac{\partial}{\partial x}\left(v \frac{\partial \varphi}{\partial x}\right)
$$


Introducing the adjoint problem (2.6) with

$$
\left(F_{\varphi}^{\prime}(\varphi, \lambda)\right)^{*} \varphi^{*}=w \frac{\partial \varphi^{*}}{\partial x}+\frac{\partial}{\partial x}\left(\lambda \frac{\partial \varphi^{*}}{\partial x}\right),
$$

and boundary conditions

$$
w \varphi^{*}+\lambda(x) \frac{\partial \varphi^{*}}{\partial x}=0, \quad x=0, \quad x=1,
$$

we get

$$
S^{\prime}(\lambda)=V_{1}\left(\lambda-\lambda_{b}\right)+\int_{0}^{T} \frac{\partial \varphi}{\partial x} \frac{\partial \varphi^{*}}{\partial x} d t
$$

and the optimality system $2.8,2.9$ and 2.10 is valid with

$$
\left(F_{\lambda}^{\prime}(\varphi, \lambda)\right)^{*} \varphi^{*}=-\int_{0}^{T} \frac{\partial \varphi}{\partial x} \frac{\partial \varphi^{*}}{\partial x} d t
$$

Due to (4.10), the covariance operator is approximately the inverse Hessian. The definition of the Hessian $H$ by $3.7,3.8$ and 3.9 involves the operators $F_{\varphi}^{\prime}(\varphi, \lambda), F_{\lambda}^{\prime}(\varphi, \lambda),\left(F_{\varphi}^{\prime}(\varphi, \lambda)\right)^{*},\left(F_{\lambda}^{\prime}(\varphi, \lambda)\right)^{*}$ defined by (4.12)-(4.14) and (4.15).

\subsection{Boundary flux estimation}

Let us consider the following evolution model:

$$
\left\{\begin{array}{l}
\frac{\partial \varphi}{\partial t}=F(\varphi)+f, \quad t \in(0, T), x \in(0,1), \\
\left.\varphi\right|_{t=0}=u, \\
-\left.k(\varphi) \frac{\partial \varphi}{\partial x}\right|_{x=0}=u_{1},\left.\quad k(\varphi) \frac{\partial \varphi}{\partial x}\right|_{x=1}=u_{2},
\end{array}\right.
$$

where $F(\varphi)$ is the $1 \mathrm{D}$ nonlinear convection-diffusion operator as follows:

$$
F(\varphi)=-\frac{\partial(w \varphi)}{\partial x}+\frac{\partial}{\partial x}\left(k(\varphi) \frac{\partial \varphi}{\partial x}\right)
$$

Above, $u_{1}$ and $u_{2}$ are the unknown boundary fluxes, $u=u(x), w=w(t, x)$ and $f=f(t, x)$ are prescribed functions, $k=k(\varphi)$ is a constitutive model for the diffusion coefficient. We consider the functional (2.2) in the form:

$$
S\left(u_{1}, u_{2}\right)=\frac{1}{2} \sum_{i=1}^{2}\left(V_{1}^{(i)}\left(u_{i}-u_{i, b}\right), u_{i}-u_{i, b}\right)_{L_{2}(0, T)}+\frac{1}{2}\left(V_{2}\left(C \varphi-\varphi_{o b s}\right), C \varphi-\varphi_{o b s}\right)_{Y_{o b s}},
$$

where $u_{i, b} \in L_{2}(0, T)$ are prescribed functions (background), $V_{1}^{(i)}: L_{2}(0, T) \rightarrow L_{2}(0, T)$ are symmetric positive definite operators, $i=1,2$. So, as a control space $Y_{p}$ (the space of parameters introduced above in Section 2), we can take $Y_{p}=L_{2}(0, T) \times L_{2}(0, T)$. Let $V_{1}: L_{2}(0, T) \times L_{2}(0, T) \rightarrow L_{2}(0, T) \times L_{2}(0, T)$ be $2 \times 2$ block-diagonal operator matrix with $V_{1}^{(1)}$ and $V_{1}^{(2)}$ as diagonal blocks. The DA problem can now be formulated as follows: find the functions $u_{1}=u_{1}(t), u_{2}=u_{2}(t), \varphi=\varphi(t, x)$ such that they satisfy (4.16), and on the set of solutions to (4.16), the functional $S\left(u_{1}, u_{2}\right)$ takes the minimum value.

Using a weak formulation of (4.16), the problem stated above may be written in the form (2.3) with $\lambda=\left(u_{1}, u_{2}\right)^{T} \in Y_{p}$, i.e. boundary conditions become a part of the operator $F$ definition. Therefore all results presented in Section 4.1 are valid in this case. However, a weak formulation is not given here because it is somewhat bulky and would only complicate the presentation. Instead, we present the auxiliary DA problem (and define all operators involved in this definition) in the usual way with the boundary conditions formulated separately.

Below we assume the solution and the input functions in (4.16) and (4.17) to be regular enough. For $v=\left(v_{1}, v_{2}\right)^{T}$ the gradient of the functional $S$ is defined by

$$
S^{\prime}\left(u_{1}, u_{2}\right) v=\sum_{i=1}^{2}\left(V_{1}^{(i)}\left(u_{i}-u_{i, b}\right), v_{i}\right)_{L_{2}(0, T)}+\left(V_{2}\left(C \varphi-\varphi_{o b s}\right), C \phi\right)_{Y_{o b s}},
$$

where $\phi$ is the solution to the problem:

$$
\left\{\begin{array}{l}
\frac{\partial \phi}{\partial t}=F^{\prime}(\varphi) \phi, \quad t \in(0, T), x \in(0,1) \\
\left.\phi\right|_{t=0}=0, \\
-\left.k(\varphi) \frac{\partial \phi}{\partial x}\right|_{x=0}=v_{1},\left.\quad k(\varphi) \frac{\partial \phi}{\partial x}\right|_{x=1}=v_{2},
\end{array}\right.
$$

and 


$$
F^{\prime}(\varphi) \phi=-\frac{\partial(w \phi)}{\partial x}+\frac{\partial^{2}(k(\varphi) \phi)}{\partial x^{2}} .
$$

Using the adjoint problem

$$
\left\{\begin{array}{l}
-\frac{\partial \varphi^{*}}{\partial t}-\left(F^{\prime}(\varphi)\right)^{*} \varphi^{*}=-C^{*} V_{2}\left(C \varphi-\varphi_{o b s}\right), \quad t \in(0, T) \\
\left.\varphi^{*}\right|_{t=T}=0, \\
w \varphi^{*}+k(\varphi) \frac{\partial \varphi^{*}}{\partial x}=0, \quad x=0, \quad x=1,
\end{array}\right.
$$

with

$$
\left(F^{\prime}(\varphi)\right)^{*} \varphi^{*}=w \frac{\partial \varphi^{*}}{\partial x}+k(\varphi) \frac{\partial^{2} \varphi^{*}}{\partial x^{2}}
$$

we get the gradient of $S$ as the vector-function:

$$
S^{\prime}\left(u_{1}, u_{2}\right)=\left(V_{1}^{(1)}\left(u_{1}-u_{1, b}\right)-\left.\varphi^{*}\right|_{x=0}, V_{1}^{(2)}\left(u_{2}-u_{2, b}\right)-\left.\varphi^{*}\right|_{x=1}\right)^{T} .
$$

Then, the optimality system involves (4.16) and (4.19), and the necessary optimality condition $S^{\prime}\left(u_{1}, u_{2}\right)=0$.

As follows from the theory developed above, the covariance operator is approximately the inverse Hessian of the following auxiliary DA problem: find $\delta u_{1}, \delta u_{2}$ and $\delta \varphi$ such that

$$
\left\{\begin{array}{l}
\frac{\partial \delta \varphi}{\partial t}-F^{\prime}(\bar{\varphi}) \delta \varphi=0, \quad t \in(0, T), \\
\left.\delta \varphi\right|_{t=0}=0, \\
-\left.k(\bar{\varphi}) \frac{\partial \delta \varphi}{\partial x}\right|_{x=0}=\delta u_{1},\left.\quad k(\bar{\varphi}) \frac{\partial \delta \varphi}{\partial x}\right|_{x=1}=\delta u_{2}, \\
S_{1}\left(\delta u_{1}, \delta u_{2}\right)=\inf _{v_{1}, v_{2}} S_{1}\left(v_{1}, v_{2}\right),
\end{array}\right.
$$

where

$$
S_{1}\left(\delta u_{1}, \delta u_{2}\right)=\frac{1}{2} \sum_{i=1}^{2}\left(V_{1}^{(i)}\left(\delta u_{i}-\xi_{i, 1}\right), \delta u_{i}-\xi_{i, 1}\right)_{L_{2}(0, T)}+\frac{1}{2}\left(V_{2}\left(C \delta \varphi-\xi_{2}\right), C \delta \varphi-\xi_{2}\right)_{Y_{o b s}} .
$$

The Hessian $H$ of the functional (4.21) is defined on $v=\left(v_{1}, v_{2}\right)^{T}$ by the successive solutions of the following problems:

$$
\begin{aligned}
& \left\{\begin{array}{l}
\frac{\partial \psi}{\partial t}-F^{\prime}(\bar{\varphi}) \psi=0, \quad t \in(0, T), \\
\left.\psi\right|_{t=0}=0, \\
-\left.k(\bar{\varphi}) \frac{\partial \psi}{\partial x}\right|_{x=0}=v_{1},\left.\quad k(\bar{\varphi}) \frac{\partial \psi}{\partial x}\right|_{x=1}=v_{2},
\end{array}\right. \\
& \left\{\begin{array}{l}
-\frac{\partial \psi^{*}}{\partial t}-\left(F^{\prime}(\bar{\varphi})\right)^{*} \psi^{*}=-C^{*} V_{2} C \psi, \quad t \in(0, T) \\
\left.\psi^{*}\right|_{t=T}=0, \\
w \psi^{*}+k(\bar{\varphi}) \frac{\partial \psi^{*}}{\partial x}=0, \quad x=0, \quad x=1,
\end{array}\right. \\
& H v=\left(V_{1}^{(1)} v_{1}-\left.\psi^{*}\right|_{x=0}, V_{1}^{(2)} v_{2}-\left.\psi^{*}\right|_{x=1}\right)^{T} .
\end{aligned}
$$

\section{Details of numerical implementation}

\subsection{Background error covariance matrix}

In the numerical implementation we deal with a finite-dimensional problem; hence we will assume that all operators in the cost functional are matrices. In order to define (2.2) and (3.25) one needs to specify the weights $V_{1}=V_{\xi_{1}}^{-1}$ and $V_{2}=V_{\xi_{2}}^{-1}$, where $V_{\xi_{1}}$ is the background error covariance matrix and $V_{\xi_{2}}$ is the observation error covariance matrix. Those two usually represent our a priori knowledge on the stochastic properties of errors.

Let us denote by $\sigma^{2}=\operatorname{diag}(V)$ the $H$-variance, $\sigma_{b}^{2}=\operatorname{diag}\left(V_{\xi_{1}}\right)$ the background error variance, and $\sigma_{o b s}^{2}=\operatorname{diag}\left(V_{\xi_{2}}\right)$ the observation error variance. We assume that the observation error values are not correlated ('white noise'), i.e. $V_{\xi_{2}}$ is a diagonal matrix. However, the same assumption about $V_{\xi_{1}}$ would be too simplistic. Therefore, the off-diagonal elements must be introduced into $V_{\xi_{1}}$.

In solving ill-posed inverse problems [23] the solution is often considered to be a smooth function which belongs to a Sobolev space of certain order, e.g. $W_{2}^{2}$. Let us assume that $\lambda(z)$ is a one-dimensional function of $z$ and introduce two weight functions $\alpha(z), \gamma(z)$ to determine the weight matrix $V_{1}$. We define a finite-difference analog of the norm in $W_{2}^{2}$ as follows:

$$
\|\lambda\|_{W_{2}^{2, m}}^{2}=\sum_{i=1}^{m} \frac{\alpha_{i}}{\sqrt{\gamma_{i}}} \lambda_{i}^{2}+\sum_{i=2}^{m-1} \frac{\alpha_{i}}{\sqrt{\gamma_{i}}}\left(\gamma_{i+1 / 2}\left(\lambda_{i+1}-\lambda_{i}\right)-\gamma_{i-1 / 2}\left(\lambda_{i}-\lambda_{i-1}\right)\right)^{2},
$$


where $\lambda_{i}, \alpha_{i}, \gamma_{i}$ are discrete values of functions $\lambda(z), \alpha(z), \gamma(z)$ at points $z_{i}, i=1, \ldots, m$ and $m$ is the number of discretization nodes.

Let us assume that the background error $\xi_{1}$ is a smooth function, i.e. it belongs to $W_{2}^{2}$. We define the symmetric weight matrix $V_{1}$ such that for any vector $\lambda$ the following relation holds:

$$
\lambda^{T} V_{1} \lambda=\|\lambda\|_{W_{2}^{2, m}}^{2}
$$

For all $\alpha_{i}>0$, the first term in (5.1) guarantees that the weight matrix $V_{1}$ is positive definite and the background error covariance matrix $V_{\xi_{1}}=V_{1}^{-1}$ can be computed. The last one is also symmetric and positive definite. It can be seen from numerical experiments that if the norm is defined by (5.1), then $\alpha(z)$ controls mainly $\sigma_{b}(z)$ and $\gamma(z)$ controls the correlation radius $r\left(z-z^{\prime}\right)$ for a wide range of $\gamma \in(0.1,100)$.

This result is illustrated in Fig. 1, where the left panel shows $\sigma_{b}^{2}(z)$ for different functions $\alpha(z)$, while $\gamma(z)$ is defined as follows:

$$
\gamma(z)=0.2+9.8(1-\cos (4 \pi z))
$$

The right panel shows the background error covariance matrix which looks identical for all functions $\alpha(z)$ considered: $\alpha=1$, $\alpha=1+3 z$, and $\alpha=\sqrt{\gamma}$. It can be seen in Fig. 1(left) that in the case $\alpha=\sqrt{\gamma}$, which corresponds to constant value of weights $\alpha_{i} / \sqrt{\gamma_{i}}$ in (5.1), the variance changes significantly with $\gamma(z)$. However, if $\alpha$ is constant (case $\alpha=1$ ), then the variance has a nearly constant value, while the changes in the correlation radius are related mainly to $\gamma(z)$. Therefore, the Eq. (5.1) can be used to generate a family of covariances such that $\alpha(z)$ and $\gamma(z)$ define mainly the variance and the correlation radius, respectively. Examples of the background error correlation functions, which correspond to different values of constant $\gamma$ for $\alpha=1$ are presented in Fig. 2, for the diffusion coefficient estimation problem (left) and for the boundary flux estimation problem (right).

In the initial-value DA problem, the background function for the subsequent DA can be computed as an optimal solution (analysis) evolved to the instant $t=T$, i.e. as $\varphi(T, x)$. Similarly, the background error covariance matrix could be computed as the evolved analysis error covariance matrix. This is possible in principle, though difficult to implement for large-scale problems. For the boundary value estimation problem such a possibility does not generally exist. Therefore, the descriptions similar to (5.1) might be a reasonable choice to define $V_{1}$ or $V_{\xi_{1}}$. Let us note that this is a typical approach for certain applications (for example, for inverse heat transfer problems [1]).

\subsection{Preconditioning the Hessian}

In [6] we have reported the numerical algorithm for computing the covariance matrix with the use of the quasi-Newton BFGS method $[4,16]$ for the case of the initial-value control problem. The same algorithm can be used in the case of parameter estimation.

Let us consider the problem (2.3). We approximate the covariance operator $V_{\delta \lambda}$ by the inverse Hessian of the auxiliary DA problem (3.24) and (3.25). The inverse Hessian (or $H$-covariance) is computed as a collateral result of the BFGS iterations in the course of solving the minimization problem (3.24) and (3.25). In order to obtain $\mathrm{H}^{-1}$ in the explicit form, the sequence of the BFGS updates must be applied to the unity vectors. For particular details of this approach we refer to [6].
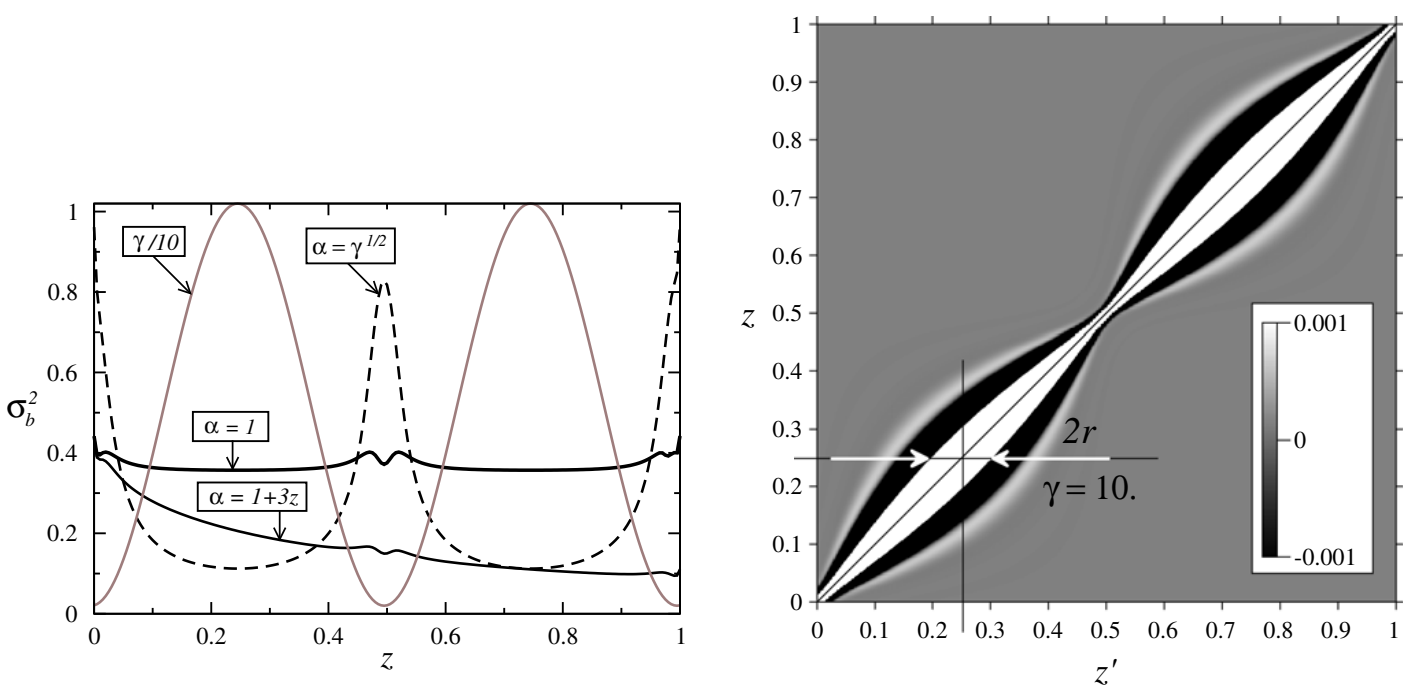

Fig. 1. Left - the background error variance $\sigma_{b}^{2}$ for different rules of change in $\alpha$, and $\gamma$ which corresponds to (5.3). Right - the background error covariance for all $\alpha$ considered. 

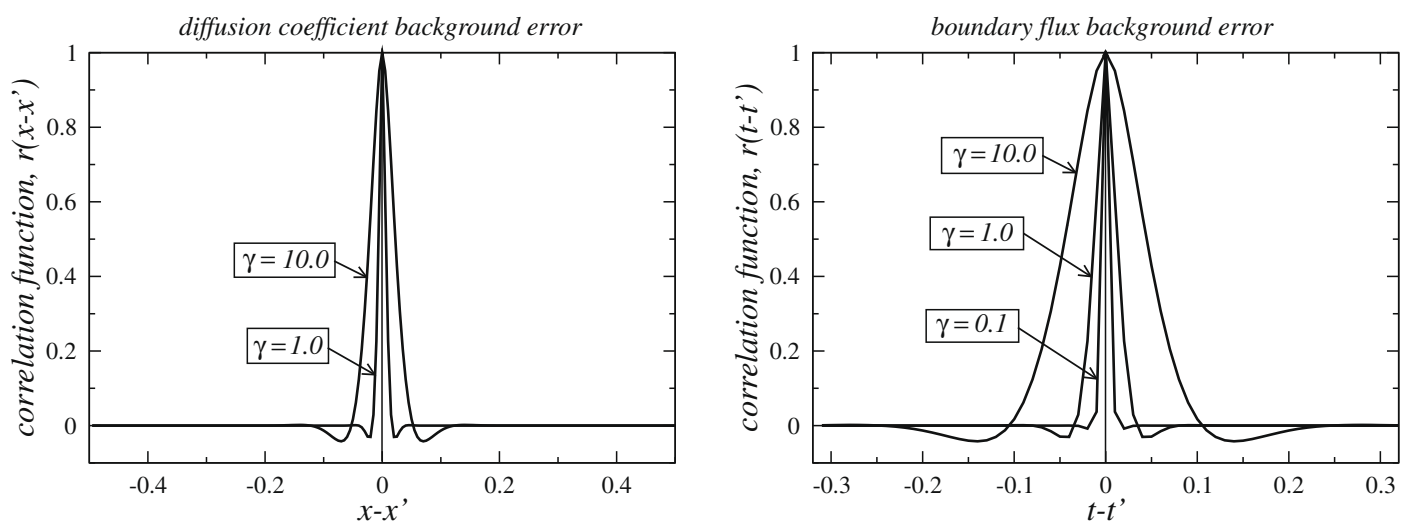

Fig. 2. Scaled correlation function of the background error $r\left(z-z^{\prime}\right)$ for different constant $\gamma$. Left - the diffusion coefficient estimation problem $\left(L=1, m=m_{x}=200\right)$. Right - the boundary flux estimation problem $\left(T=0.064, m=m_{t}=64\right)$.

The process of building the inverse Hessian by the BFGS algorithm can be accelerated by using a preconditioning. Usually, a preconditioning is aimed to accelerate the convergence rate of a minimization procedure. However, this is a different task. For example, the minimization procedure may converge long before any sensible approximation of the inverse Hessian is built. Here we assume that the preconditioned Hessian would have much fewer eigenvalues remote from 1 and, therefore, require fewer quasi-Newton updates to be represented.

Since $H$ is self-adjoint, we must consider a preconditioned Hessian in a symmetric form, for example:

$$
\widetilde{H}=\left(B^{-1}\right)^{*} H B^{-1},
$$

with some operator $B: Y_{p} \rightarrow Y_{p}$. We can prove that the operator $\widetilde{H}$ is the Hessian of the following modified auxiliary DA problem: find $\delta \lambda$ and $\delta \varphi$ such that

$$
\left\{\begin{array}{l}
\frac{\partial \delta \varphi}{\partial t}-F_{\varphi}^{\prime}(\bar{\varphi}, \bar{\lambda}) \delta \varphi=F_{\lambda}^{\prime}(\bar{\varphi}, \bar{\lambda}) B^{-1} \delta \lambda, \quad t \in(0, T) \\
\left.\delta \varphi\right|_{t=0}=0 \\
S_{2}(\delta \lambda)=\inf _{v \in Y_{p}} S_{2}(v)
\end{array}\right.
$$

where

$$
S_{2}(\delta \lambda)=\frac{1}{2}\left(V_{1} B^{-1}\left(\delta \lambda-\xi_{1}\right), B^{-1}\left(\delta \lambda-\xi_{1}\right)\right)_{Y_{p}}+\frac{1}{2}\left(V_{2}\left(C \delta \varphi-\xi_{2}\right), C \delta \varphi-\xi_{2}\right)_{Y_{o b s}} .
$$

Therefore, one may use the BFGS algorithm to solve the minimization problem (5.5) and (5.6) and find $(\widetilde{H})^{-1}$. After that, having $(\widetilde{H})^{-1}$, one can easily recover $H^{-1}$ using the formula

$$
H^{-1}=B^{-1}(\widetilde{H})^{-1}\left(B^{-1}\right)^{*} \text {. }
$$

For the boundary flux estimation problem (Section 4.3) the modified auxiliary problem (5.5) and (5.6) reads as follows: find $\delta u_{1}, \delta u_{2}$ and $\delta \varphi$ such that

$$
\left\{\begin{array}{l}
\frac{\partial \delta \varphi}{\partial t}-F^{\prime}(\bar{\varphi}) \delta \varphi=0, \quad t \in(0, T) \\
\left.\delta \varphi\right|_{t=0}=0 \\
-\left.k(\bar{\varphi}) \frac{\partial \delta \varphi}{\partial x}\right|_{x=0}=B_{1}^{-1} \delta u_{1},\left.\quad k(\bar{\varphi}) \frac{\partial \delta \varphi}{\partial x}\right|_{x=1}=B_{2}^{-1} \delta u_{2} \\
S_{2}\left(\delta u_{1}, \delta u_{2}\right)=\inf _{v_{1}, v_{2}} S_{2}\left(v_{1}, v_{2}\right)
\end{array}\right.
$$

where

$$
S_{2}\left(\delta u_{1}, \delta u_{2}\right)=\frac{1}{2} \sum_{i=1}^{2}\left(V_{1}^{(i)} B_{i}^{-1}\left(\delta u_{i}-\xi_{i, 1}\right), B_{i}^{-1}\left(\delta u_{i}-\xi_{i, 1}\right)\right)_{L_{2}(0, T)}+\frac{1}{2}\left(V_{2}\left(C \delta \varphi-\xi_{2}\right), C \delta \varphi-\xi_{2}\right)_{Y_{o b s}} .
$$

One can recover $H^{-1}$ by the formula (5.7), where $B$ is $2 \times 2$ block-diagonal matrix with $B_{1}$ and $B_{2}$ as blocks.

An important issue is how to construct the operator $B^{-1}$. Usually one tries to take $B^{-1}$ in such a way that the spectrum of the preconditioned Hessian $\widetilde{H}=\left(B^{-1}\right)^{*} H B^{-1}$ is clustered around 1 . This means that the majority of eigenvalues of $\widetilde{H}$ are equal or close to 1 . Theoretically, the best choice of $B^{-1}$ is such that $\widetilde{H}$ is the identity operator. Thus, one should achieve $B^{-1}\left(B^{-1}\right)^{*} \approx H^{-1}$ or $B^{*} B \approx H$. One possibility to construct $B^{-1}$ is to consider an approximation $H_{a} \approx H$. If we compute the Cholesky factorization for $H_{a}^{-1}$ in the form $H_{a}^{-1}=L L^{*}$, then we can take $B^{-1}=L$. Sometimes, it is beneficial to compute the Cholesky factorization for $H_{a}$ itself, i.e. $H_{a}=L L^{*}$, then we can take $B^{-1}=\left(L^{*}\right)^{-1}$. 
In this paper we assume that $H_{a}=V_{1}$. This choice of $H_{a}$ corresponds to the 'first level' preconditioning commonly used in variational DA [3,5]. In the $1 \mathrm{D}$ case, the weight matrix $V_{1}$ defined by (5.1) and (5.2) is a five-diagonal banded matrix. For a given $V_{1}$ we compute the Cholesky factorization $V_{1}=L L^{*}$, where $L$ is the lower triangular factor of $V_{1}$. Then, the product $B^{-1} v$ (required in Eqs. 5.5, 5.6 and 5.7) can be obtained by the backward substitution sweep involving $L^{*}$, while $\left(B^{-1}\right)^{*} v($ required in (5.7)) - by the forward substitution sweep involving $L$. With this arrangement the matrix $V_{\xi_{1}}$ is never computed. Other possible approaches to Hessian preconditioning are considered in [5,24,26]. Let us emphasize that the development of an efficient preconditioner would be the key implementation issue for the method proposed.

\subsection{Additional implementation details}

As a model for numerical implementation we consider the 1D convection-diffusion equation. We use the implicit time discretization as follows

$$
\frac{\varphi^{i}-\varphi^{i-1}}{h_{t}}+\frac{\partial\left(w \varphi^{i}\right)}{\partial x}-\frac{\partial}{\partial x}\left(k\left(\varphi^{i}\right) \frac{\partial \varphi^{i}}{\partial x}\right)=0, \quad i \in\left(1, \ldots, m_{t}\right), x \in(0,1)
$$

where $i=1, \ldots, m_{t}$ is the time integration index, $h_{t}=T / m_{t}$ is a time step. The spatial operator is discretized on a uniform mesh ( $h_{x}=1 / m_{x}$ is the spatial discretization step, $m_{x}$ is the total number of mesh nodes) using the 'power law' first-order scheme as described in [15].

When the boundary flux estimation problem is considered (Section 4.3), the model equation is nonlinear $(k=k(\varphi))$. In this case for each time step we perform nonlinear iterations, assuming initially that $k\left(\varphi^{i}\right)=k\left(\varphi^{i-1}\right)$, and keep iterating until (5.10) is satisfied (i.e. the norm of the left-hand side in (5.10) becomes smaller than a threshold $\epsilon_{1}=10^{-12} \sqrt{m_{x}}$ ). For the parameter estimation problem (Section 4.2) we assume that the diffusion coefficient does not depend on the solution $\varphi$, but is a function of $x$, i.e. $k=k(x)$. Let us notice that even though the model equation becomes linear in this case, the parameter estimation problem remains nonlinear (that can be seen from (4.15)).

Let us note that the auxiliary DA problem (3.24) and (3.25) includes the TLM of the original evolution problem. In order to solve the minimization problem (3.24) and (3.25) using the BFGS algorithm one also needs the adjoint model that computes the gradient of (3.25) with respect to the unknown parameters. Both in [6] and in the present paper we use the TLM and adjoint models generated by means of Automatic Differentiation (AD) [8]. The TLM and adjoint models produced in this way are known as consistent models. We stress that the use of consistent models is essential to obtain the $H$-covariance. However, if the TLM code is produced by means of AD, it could be difficult to separate manually the part of the code which computes the solution of the original evolution problem and the part which computes the solution of the TLM. With this arrangement the original nonlinear problem has to be solved as many times as the TLM whereas it should be solved only once. Let us notice that for solving the auxiliary problem (3.24) and (3.25) the consistency between the TLM and the adjoint models is all that is required. Therefore, the compromise approach would be: (a) derive analytically and separately implement the TLM, and (b) generate the adjoint model by means of AD, using the TLM source code as the input for the AD engine.

In numerical experiments, the $H$-covariance matrix $V$ will be compared with the ensemble covariance matrix $\widehat{V}$, obtained by the fully nonlinear ensemble method. This method is presented in detail in [6]. Here we would only mention that this method allows the covariance matrix to be estimated without any linearization involved, and therefore is considered for the verification purpose. The ensemble size $M=400$ is being used in all ensemble computations presented in this paper. This size has been chosen such that the sampling error is noticeably smaller $(\approx 10 \%)$ than the optimal solution error.

\section{Numerical results}

We mentioned already that DA allows the uncertainty in model parameters/controls to be reduced. The background error covariance matrix $V_{\xi_{1}}$ (a priori covariance matrix) is a measure of uncertainty in model parameters before DA. The variance $\sigma_{b}^{2}$ can be considered as the original uncertainty magnitude. The (optimal solution error) covariance matrix $V_{\delta \lambda}$ (a posteriori covariance matrix) is a measure of uncertainty in the sought parameters after DA and the variance $\sigma_{\delta \lambda}^{2}$ is the uncertainty magnitude after DA, respectively. Let us introduce the function

$$
\zeta(z)=\sigma^{2}(z) / \sigma_{b}^{2}(z), \quad z \in[0,1]
$$

where $\sigma^{2}(z)$ is the $H$-variance. The function $\zeta$ quantifies the 'usefulness' of observation data in terms of reducing the original uncertainty magnitude. Let us note that $0<\zeta<1: \zeta \rightarrow 1$ means that the efficiency of DA decreases (i.e. the original uncertainty is less and less affected), $\zeta \rightarrow 0$ means that DA is increasingly efficient. Below, the results of most numerical experiments are presented in terms of $\zeta$. In addition to $\zeta$ obtained by the BFGS, we present $\hat{\zeta}$ which is obtained by the ensemble method. These two must be in a proper agreement if the linearization error is small enough and the ensemble size $M$ is sufficiently large. 


\subsection{Distributed coefficient estimation}

Here we refer to the diffusion parameter estimation problem stated in Section 4.2. In this problem one tries to estimate the unknown diffusion coefficient $k(x), x \in(0,1)$ using a set of incomplete observations of the field $\varphi(t, x)$ which evolves from the known initial state $u$. Let us note that the evolution Eq. (4.11) is now linear, however the parameter estimation problem is always nonlinear. We consider two cases: the convection-dominated case $\left(w=10, k=0.01, P e=w / k=10^{3}\right)$, and the pure diffusion case $(w=0, k=0.1)$. In the first case the initial state is the step-function

$$
u=\left\{\begin{array}{ll}
1, & x \leqslant 0.1 \\
0, & x>0.1
\end{array},\right.
$$

in the second case it is defined by the formula

$$
u=0.5(1+\cos (4 \pi x)) \text {. }
$$

The 'true' field $\bar{\varphi}(t, x)$ for both cases is presented in Fig. 3(left) and Fig. 3(right), respectively. In geophysics, the convectiondominated problems are usual in meteorology and surface-water applications. For example, Fig. 3(left) may represent a heat wave propagation. Even though the (eddy) diffusion could be relatively small, it is an important parameter that defines the front dissipation rate. The diffusion-dominated problems arise in groundwater [20] and oil-reservoir modelling.

In order to compute the $H$-covariance $V$ we solve the modified auxiliary DA problem (5.5) and (5.6) with $\lambda=k$ by the BFGS algorithm, then retrieve $V=H^{-1}$ using (5.7). The discretization parameters for the numerical model are: $m_{x}=200$, $h_{x}=0.05, m_{t}=128, T=0.064, h_{t}=0.005$.

\subsubsection{Convection-dominated evolution model}

In this part we consider the observation scheme which consists of five sensors located in the middle of the computational domain at the points $x=0.4,0.45,0.5,0.55,0.6$; the observation error variance is constant in $x$ with $\sigma_{o b s}=3 \times 10^{-4}$. Since the diffusion coefficient is always positive, the function $\alpha(x)$ (which largely defines $\sigma_{b}(x)$ ) must be considered such that $3 \sigma_{b}(x)<\bar{k}(x), \forall x$. We chose $\alpha(x)$ to satisfy the condition $3 \sigma_{b}(x) \approx \bar{k}(x)$. This allows us to apply the largest possible background error $\xi_{1}$, while keeping $k_{b}=\bar{k}+\xi_{1}$ positive (and therefore physically meaningful) in ensemble computations. This is a limitation of the theory presented in this paper that results from the assumption of the Gaussian (i.e. symmetric) distribution of the background error and could be particularly noticeable for small $\bar{k}(x)$.

In the first example we consider $\bar{k}(x)=0.01, w=10, \gamma(x)=10$, and $\alpha(x)=4 \times 10^{4}$. The result obtained by the BFGS (the function $\zeta(x),(6.1)$ ) is presented in Fig. 4(left) in bold solid line. One can see that the behaviour of $\zeta$ is relatively simple and generally resembles the behaviour of the variance in the initial-value control problem ([6]). The minima of $\zeta$ are located in the vicinity of sensors. Between the sensors $\zeta$ grows to a level which depends mainly on the background error correlation radius controlled by $\gamma(x)$. Outside the domain covered by sensors $\zeta$ grows approaching 1 , even though there is an intermediate level of $\zeta<1$ in the upstream direction. For the same conditions we compute the ensemble $\hat{\zeta}$ (presented in the marked line). One can notice that $\hat{\zeta}$ is in a good agreement with $\zeta$ obtained by the BFGS, particularly within the area where sensors dominate the look of $\zeta$. The $H$-covariance matrix $V$ obtained by the BFGS and $\hat{V}$ obtained by the ensemble method are presented in Fig. 5(left) and Fig. 5(right), respectively.

In order to compare cases with different $k(x)$ we consider the following functions: $k(x)=0.1, w=10, \gamma(x)=10$, and $\alpha(x)=4 \times 10^{2}$. The result obtained by the BFGS is presented in Fig. 4(left) in faint solid line. We note that now the structure of $\zeta$ is even simpler than before and that the larger $k(x)$ can be better estimated (with the same $\sigma_{\text {obs }}$ ).

In the second example presented in Fig. 4(right) we compare $\zeta$ computed with $\gamma(x)=10$ (in bold solid line) and with the variable $\gamma$ defined by (5.3)(in faint solid line). One can notice a very significant difference between these cases. This example underlines the crucial role of the background error correlation radius (controlled by $\gamma$ ) in computing the covariance.
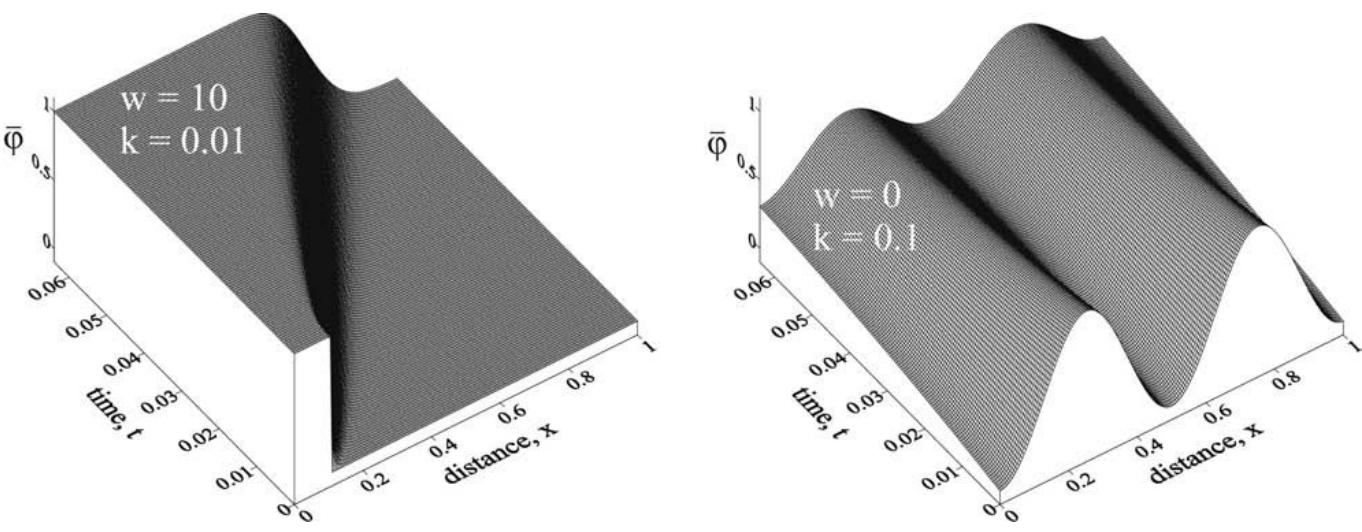

Fig. 3. -Field evolution for convection-dominated problem (left) and pure diffusion problem (right). 

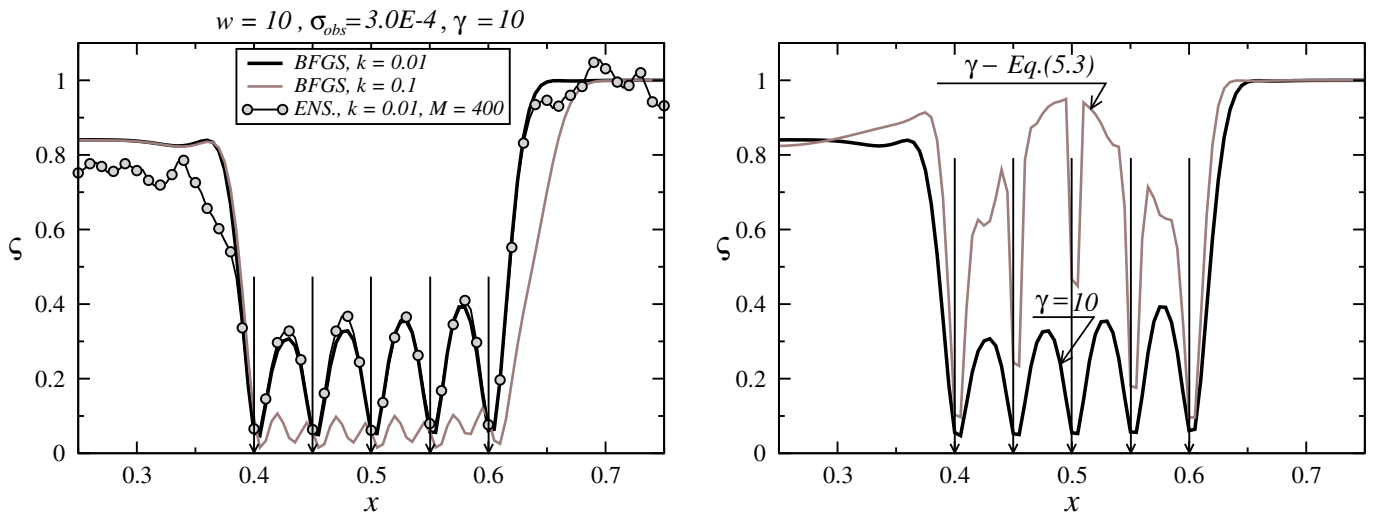

Fig. 4. Left $-\zeta$ and $\hat{\zeta}$ for $k=0.01$ and $\zeta$ for $k=0.1$. Right $-\zeta$ for varying $\gamma$ and for constant $\gamma=10$.
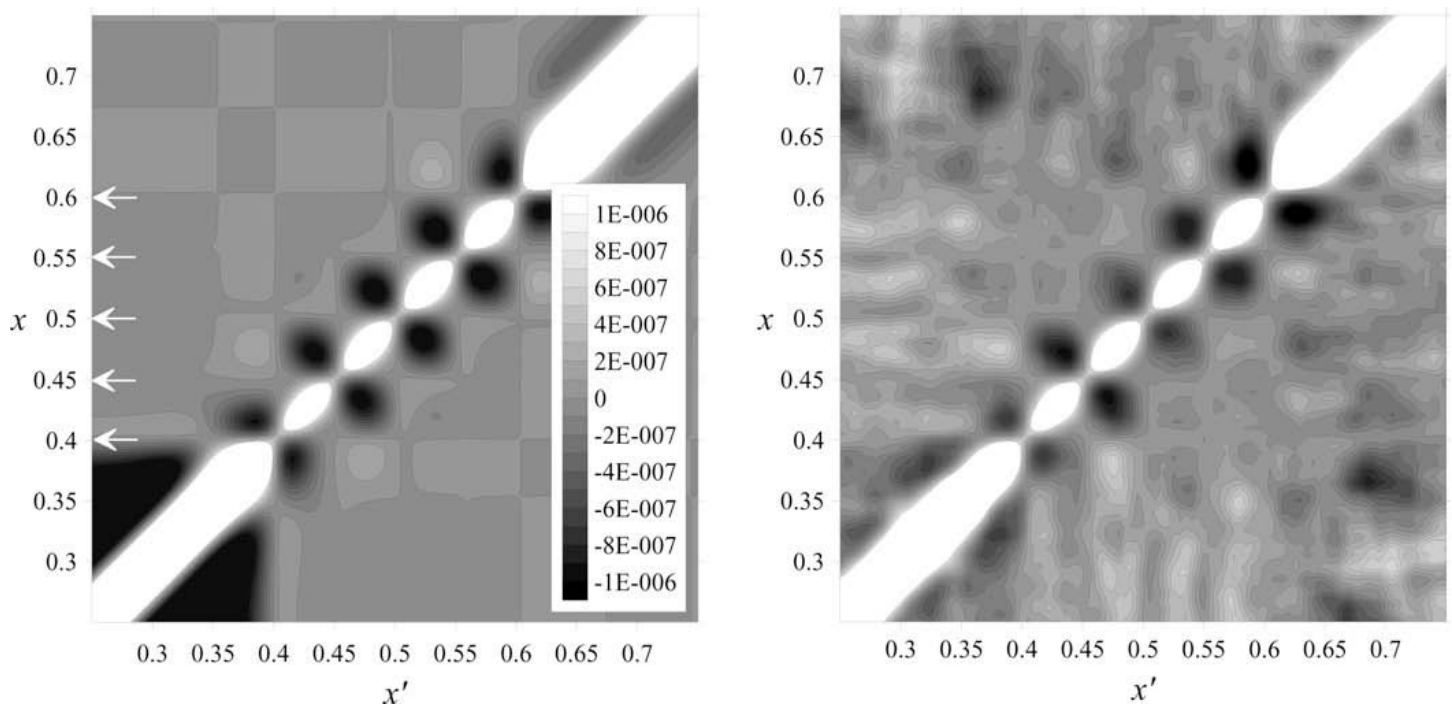

Fig. 5. Diffusion coefficient estimation problem. Left - H-covariance. Right - ensemble covariance.
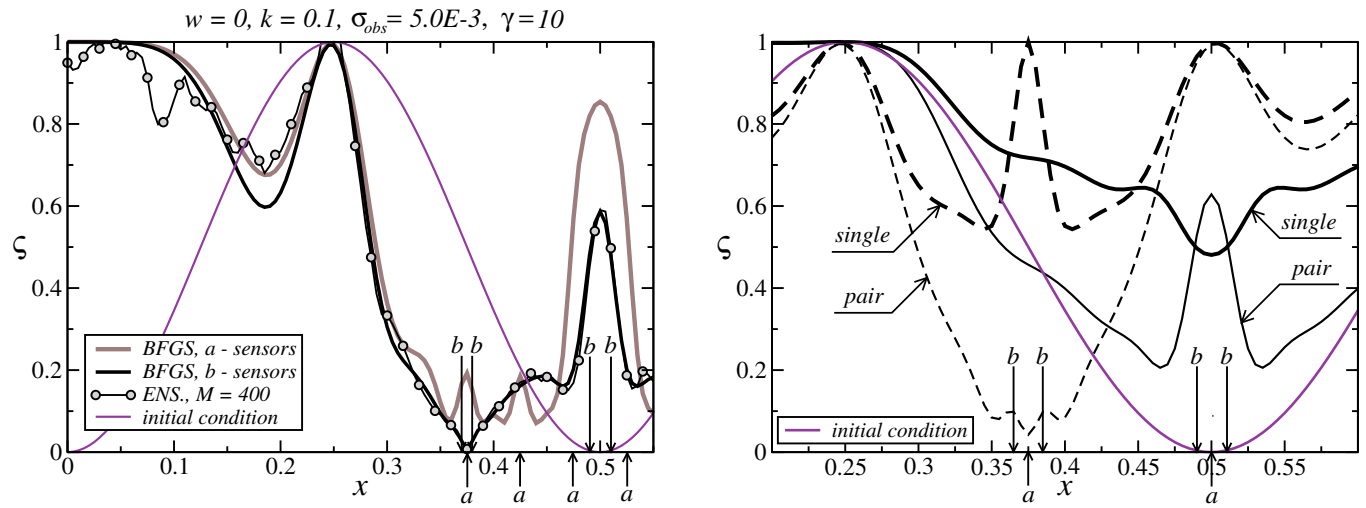

Fig. 6. $H$-variances. Left $-\zeta$ for 2 observation schemes and $\hat{\zeta}$. Right $-\zeta$ for a single sensor versus $\zeta$ for a pair of sensors.

\subsubsection{Pure diffusion evolution model}

In this part we refer to Fig. 6(left). We consider two observation schemes. Each scheme consists of six sensors located in the middle of the spatial domain, however the sensors are located differently. In both cases the observation error variance is constant in $x$ with $\sigma_{o b s}=5 \times 10^{-3}$. In the first case the sensors are evenly distributed (locations are shown by arrows marked $a$ ), the corresponding $\zeta(x)$ is presented in faint solid line. In the second case the sensors are put in pairs (locations are shown 

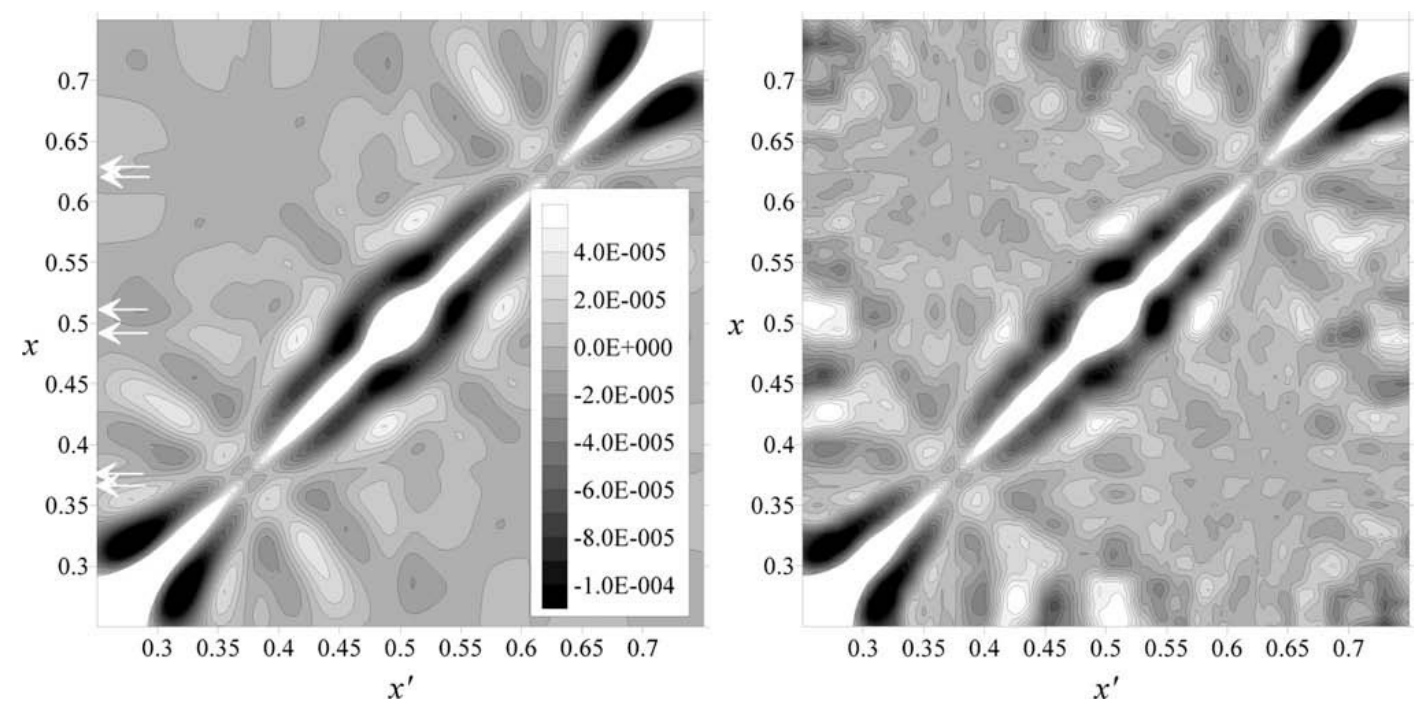

Fig. 7. Diffusion coefficient estimation problem. Left - H-covariance. Right - ensemble covariance.

by arrows marked $b$ ), the result is presented in bold solid line. We notice that in the second case the function $\zeta(x=0.375)$ is smaller by an order of magnitude than in the first case. For the second case we also present the ensemble $\hat{\zeta}$ by the marked line. One can see a very good agreement between $\zeta$ and $\hat{\zeta}$, particularly in the area covered by sensors. For this case the $H-$ covariance $V$ and the ensemble covariance $\widehat{V}$ are presented in Fig. 7(left) and Fig. 7(right), respectively.

In the case of pure diffusion the behaviour of $\zeta$ is more complex than in the convection-dominated case. This can be explained by considering the expression (4.14), for example. We notice that the function $\partial \varphi^{*} / \partial x$, which is responsible for delivering information from the sensors via the adjoint variable, is multiplied by $\partial \varphi / \partial x$. Because of that, in the areas with small field gradients the gradient of the cost function $S^{\prime}(\lambda)$ is dominated by the background term. Due to the symmetric nature of the diffusion process, the extremum points in the initial state do not change their original location (see e.g. Fig. 3(right)), i.e. the areas with small and big gradients remain at certain locations.

Below we refer to Fig. 6(right). Let us assume that the observation system includes a single sensor and the initial condition satisfies to (6.2). First we put the sensor at the location $x=0.5$, where the field gradient tends to zero, but the field value $\varphi(t, x)$ changes most significantly. Next we put the sensor at the location $x=0.375$, where the field gradient reaches its maximal value, however the field value remains constant $\varphi(t, x)=0.5$. The results (function $\zeta(x)$ ) are presented in bold solid and bold dashed lines, respectively. In the first case $(x=0.5)$, as expected, no significant reduction in $\zeta$ is achieved. In the second case $(x=0.375)$ the result is actually even worse (around the sensor location). The point is that the constant field value measured by the sensor may correspond to any initial condition that satisfies $u=0.5(1+\cos (2 n \pi x)), n=2,3, \ldots$, which means that without the background term the problem has no unique solution. This analysis leads to the conclusion that in given circumstances it might be beneficial to observe the field gradient, rather than the field value. In order to support this idea, instead of a single sensor at $x=0.375$, we put a pair of sensors located closely $\left(x_{1}=0.365\right.$ and $\left.x_{2}=0.385\right)$. The corresponding $\zeta$ is presented in Fig. 6(right) by a thin dashed line. One can see that we have achieved a drastic decrease in $\zeta$. Finally, we put a pair of sensors $\left(x_{1}=0.49\right.$ and $\left.x_{2}=0.51\right)$ instead of a single sensor at $x=0.5$. One can see that the same decrease in $\zeta$ is not achieved. This must be expected since the field gradient at $x=0.5$ is equal to zero. A practical conclusion from these numerical experiments is that at the areas where the field gradient is big enough one may use a pair of closely located sensors to catch the field gradient. In this case the quality of the diffusion coefficient estimation can be greatly improved. A simple structure of $\zeta$ in the convection-dominated problem considered above can now be explained. The reason is that the convection moves the field pattern (front) across the domain, therefore a similar field and field gradient values are supplied to each sensor at some stage.

\subsection{Boundary flux estimation problem}

Here we refer to the parameter estimation problem stated in Section 4.3. For a trivial initial condition $u=0$, one looks to estimate unknown boundary fluxes $u_{1}$ and $u_{2}$ using a set of incomplete observations $\varphi_{o b s}$. The observation scheme includes three sensors located at points $x=0.2,0.5,0.8$. The evolution Eq. (4.16) is nonlinear because the diffusion coefficient $k(\varphi)$ depends on $\varphi$. In order to set up tests we consider two cases of $k(\varphi)$ as shown in Fig. 8(left).

The diffusion coefficient $k(\varphi)$ varies from the level $k_{1}$ to the level $k_{2}\left(k_{2}>k_{1}\right.$ for case $I$ and $k_{2}<k_{1}$ for case II) within the interval $\left[\varphi_{0}-\Delta, \varphi_{0}+\Delta\right]$ subjected to the rule as follows:

$$
k(\varphi)=\frac{k_{1}+k_{2}}{2}-\frac{k_{1}-k_{2}}{2} \sin \left(\pi\left(\varphi-\varphi_{0}\right) /(2 \Delta)\right) .
$$



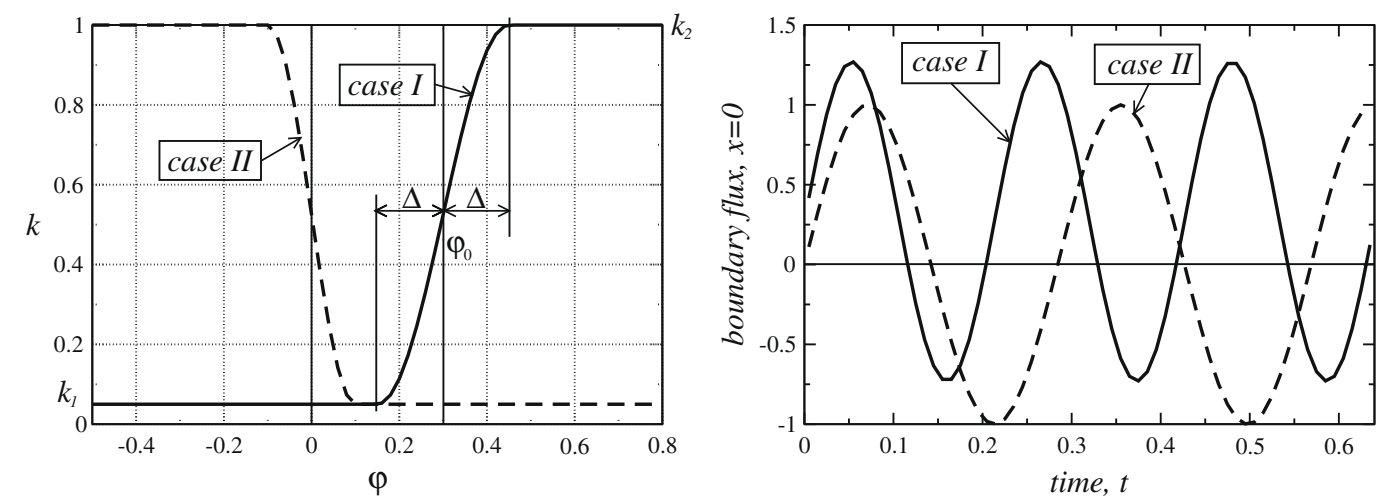

Fig. 8. Left - diffusion coefficient $k(\varphi)$. Right - 'true' boundary conditions.

We can control the degree of nonlinearity (up to a nearly discontinuous $k(\varphi)$ ) by changing parameters $\Delta$ and $k_{1}, k_{2}$. In case $I$ we choose $\varphi_{0}=0.3, k_{1}=0.05, k_{2}=1.0, \Delta=0.15$, in case $I I-\varphi_{0}=0.0, k_{1}=1.0, k_{2}=0.05, \Delta=0.1$. The discretization parameters for the numerical model are: $m_{x}=200, h_{x}=0.05, m_{t}=64, T=0.64, h_{t}=0.01$. The 'true value' of the driving boundary condition $\bar{u}_{1}$ for two cases under consideration are presented in Fig. 8(right), while $\bar{u}_{2}=0$.

\subsubsection{Inflow driving boundary}

A distinguishing feature of this case is that the driving boundary at $x=0$ is the inflow boundary (i.e. $w>0(|w|=2)$ ), therefore the boundary perturbations propagate far enough into the domain. The field variable $\bar{\varphi}(x, t)$ for this case is presented in Fig. 9(left), the diffusion coefficient $k(\bar{\varphi}(x, t))$ in Fig. 9(right). We compute the $H$-covariance and the ensemble
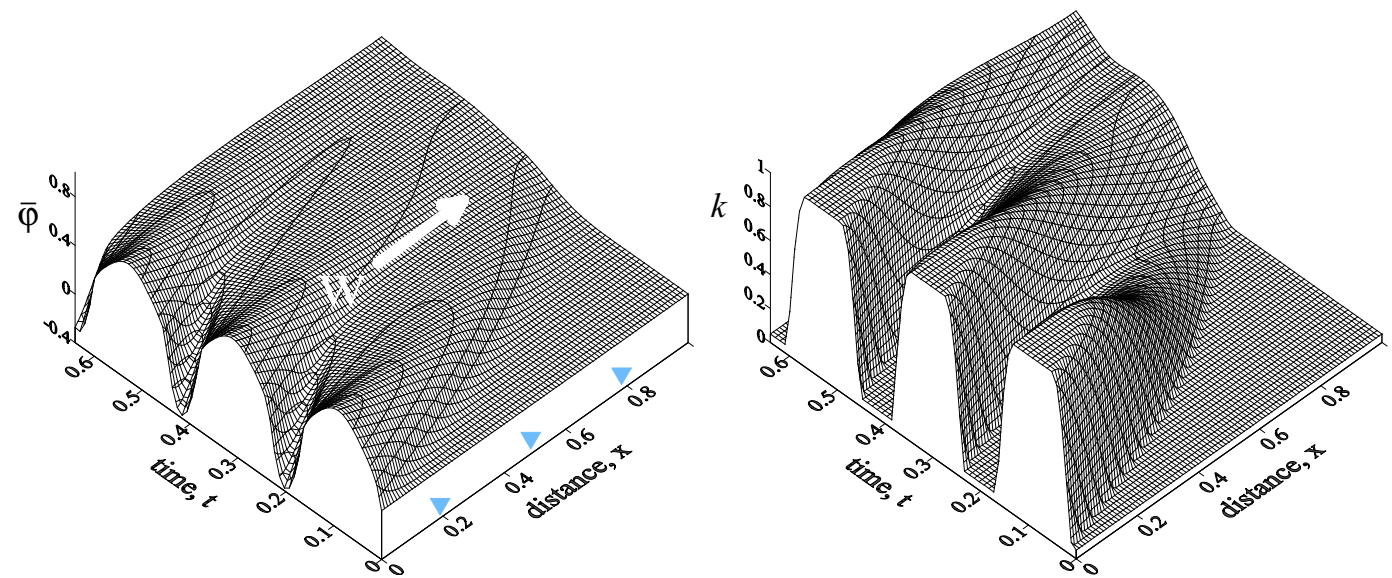

Fig. 9. Left - 'true' field $\bar{\varphi}(t, x)$. Right - diffusion coefficient $k(\bar{\varphi}(t, x))$.
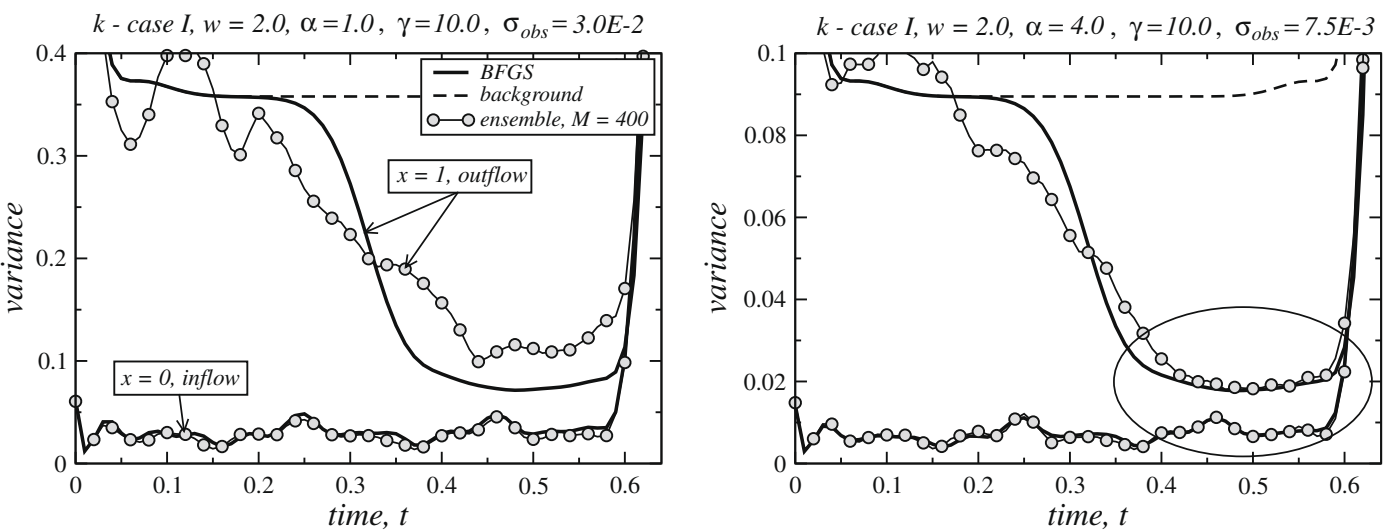

Fig. 10. Left - background, $H$ - and ensemble variances for larger errors $\xi_{i}$. Right - variances for smaller errors $\xi_{i}$. 
covariance assuming the following properties of errors $\xi_{i}: \alpha=1.0, \gamma=10.0, \sigma_{o b s}=0.03$. The corresponding variances are presented in Fig. 10(left).

The first thing to be noticed in this figure is that the variance of the unknown flux $u_{1}$ at the inflow boundary $x=0$ (solid line) is much smaller than the background variance (dashed line), which means it can be well identified almost everywhere, excluding a 'blind spot' interval $(T-\delta t, T)$. The match between the $H$-variance and the ensemble variance is good. This result is trivial and could easily be predicted from a qualitative analysis.

An unexpected conclusion is however that the flux $u_{2}$ at the outflow boundary $x=1$ can also be partially identified $(t \in(0.38,0.6))$. This 'identifiable' part of the boundary condition $u_{2}$ corresponds to a larger value of the diffusion coefficient $k(\varphi)$ (Fig. 9(right)), which is due to the nonlinearity. This behaviour would be difficult to expect without computations.

Let us notice that the match between the $H$-variance and the ensemble variance for $u_{2}$ is not particularly good. Indeed, the $H$-covariance is an approximation of the actual covariance which relies on the linearized error evolution model (TLM). In ([6]) we emphasize that the tangent linear hypothesis is a local sufficient condition. Because of that, the $H$-covariance could be a good approximation of the covariance far beyond the validity of this condition. The accuracy of the linearization depends on two factors: a) degree of the nonlinearity; b) magnitude of errors $\xi_{i}$. This means that the estimation/control problem could be extremely nonlinear, yet the linearization would be accurate if the magnitude of errors is sufficiently small. In the case considered above the magnitude of the background error was noticeably larger than the unknown fluxes themselves.

To illustrate this point we consider another case with the errors magnitude being $1 / 4$ of those in the previous case: $\alpha=4.0, \gamma=10.0, \sigma_{o b s}=0.0075$. The variances for this case are presented in Fig. 10 (right). We can see that the $H$ - and the ensemble variances are now in a much better agreement, particularly in the area highlighted by the ellipse. Therefore, for smaller errors, the $H$-covariance is again a good approximation of the covariance.

Next we analyse how the $H$-covariance $V$ depends on the correlation radius of the background error $r\left(t-t^{\prime}\right)$, which is controlled by $\gamma$ (Fig. 2(right)). The function $\zeta=\zeta(t)$ for different $\gamma$ is presented in Fig. 11 for $u_{1}$ (left) and for $u_{2}$ (right). In [6] we mentioned that $\gamma$ is a crucial parameter which defines the $H$-covariance. The same conclusions can be drawn from Fig. 11 . We can see that for a weakly correlated background error $\gamma=0.1$, the function $\zeta$ is close to 1 even for the inflow boundary $x=0$, Fig. 11(left). As $\gamma$ grows, $\zeta$ becomes smaller, i.e. the efficiency of DA increases. This example shows that if we specify the background error correlation radius wrongly (which may happen since $\gamma$ is often a priori defined function!), then the covariance
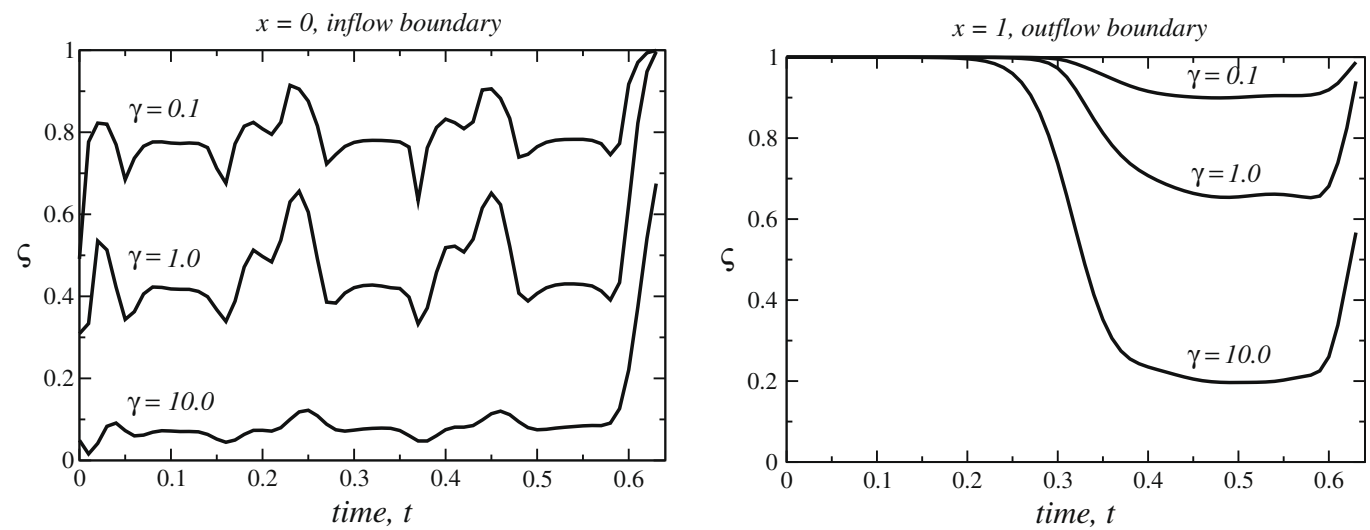

Fig. 11. Left $-\zeta$ at inflow boundary $x=0$ for different $\gamma$. Right $-\zeta$ at outflow boundary $x=1$.
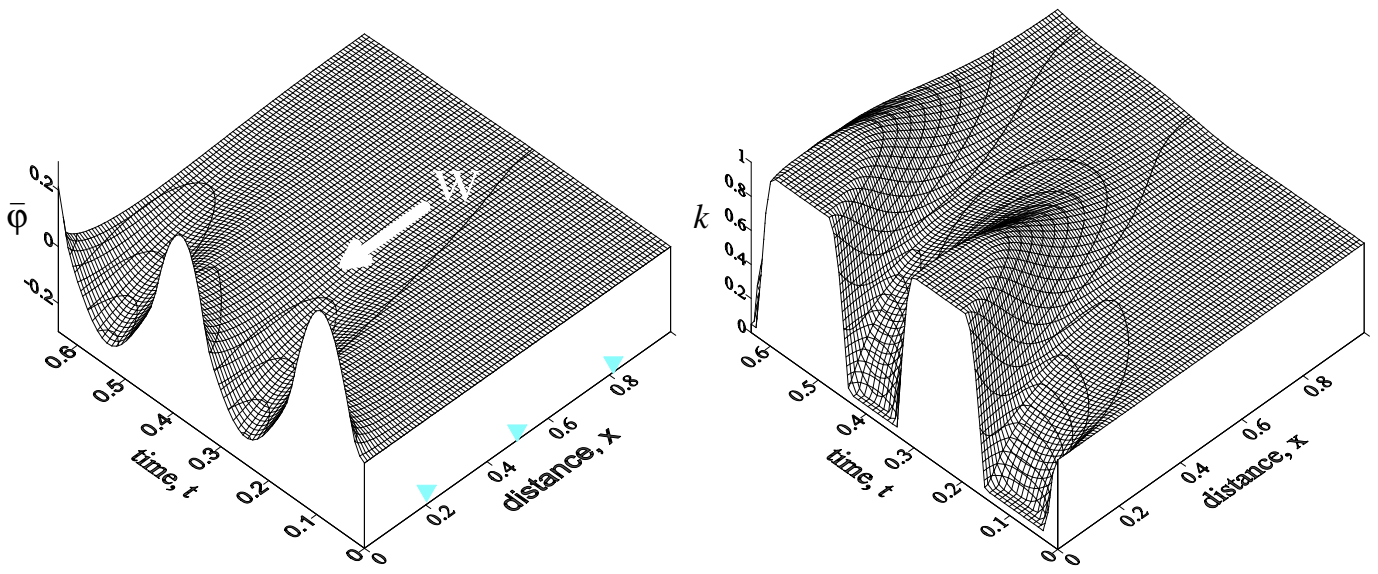

Fig. 12. Left - 'true' field $\bar{\varphi}(t, x)$. Right - diffusion coefficient $k(\bar{\varphi}(t, x))$. 
estimate (both $H$-covariance and the ensemble covariance) could be wrong. One could possibly state that any discussion on the 'linearization error issue' is irrelevant unless we are certain about the background error correlation function.

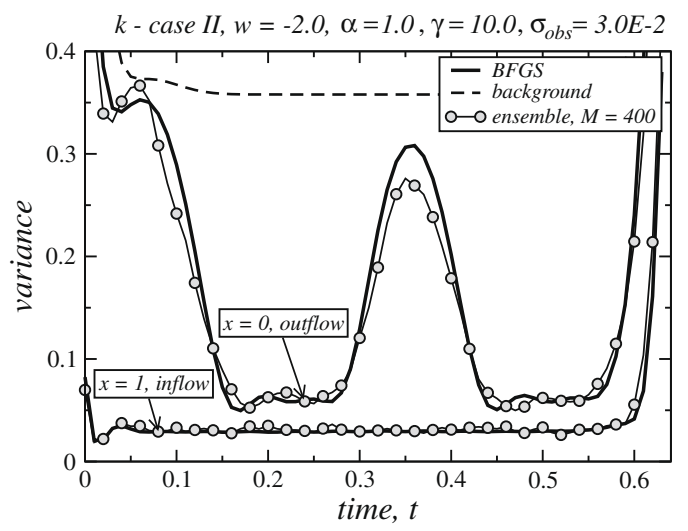

Fig. 13. Background, $\mathrm{H}-$ and ensemble variances.
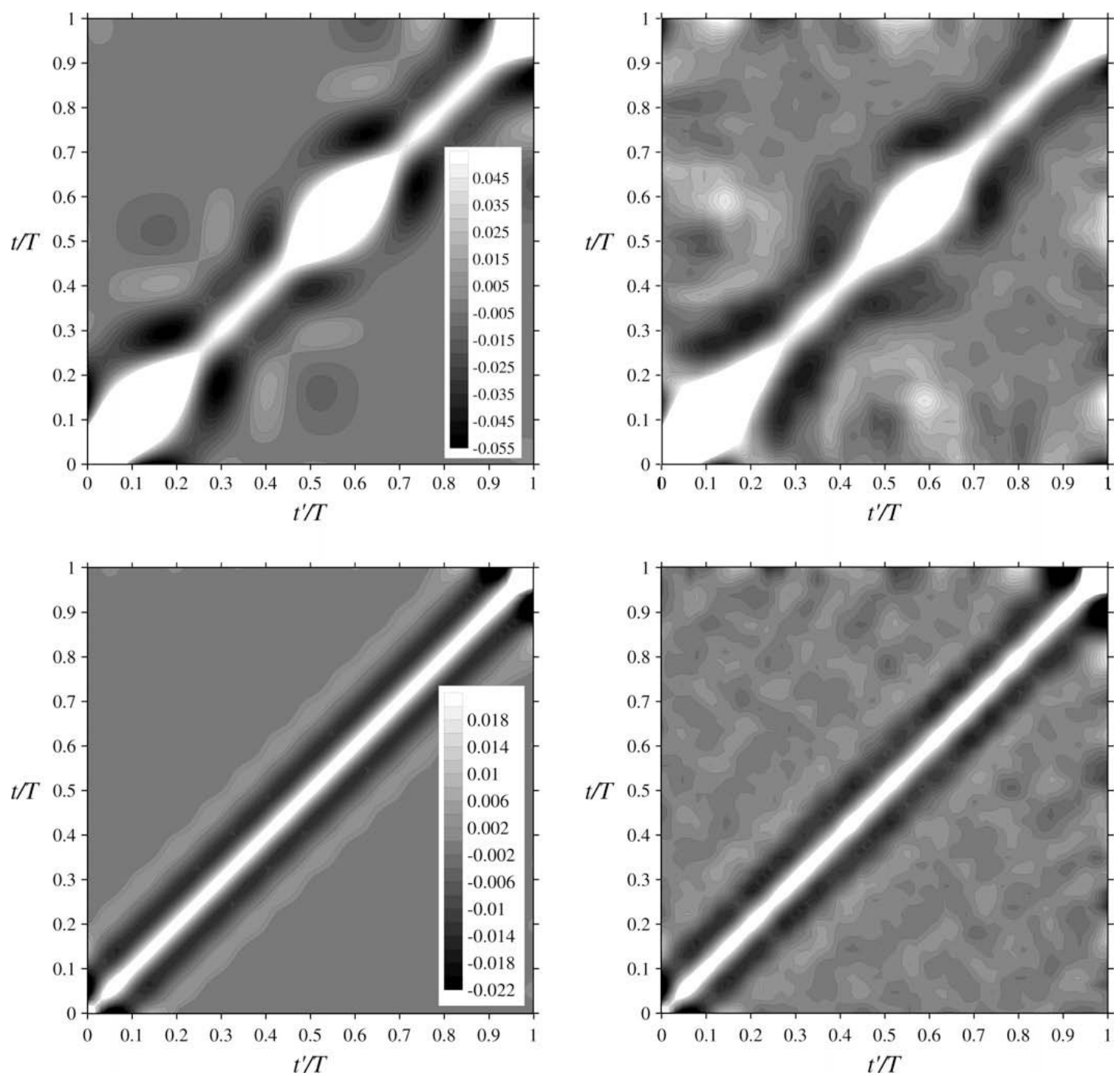

Fig. 14. Left, upper - H-covariance for $\delta u_{1}$. Right, upper - ensemble covariance for $\delta u_{1}$. Left, lower - H-covariance for $\delta u_{2}$. Right, lower - ensemble covariance for $\delta u_{2}$. 

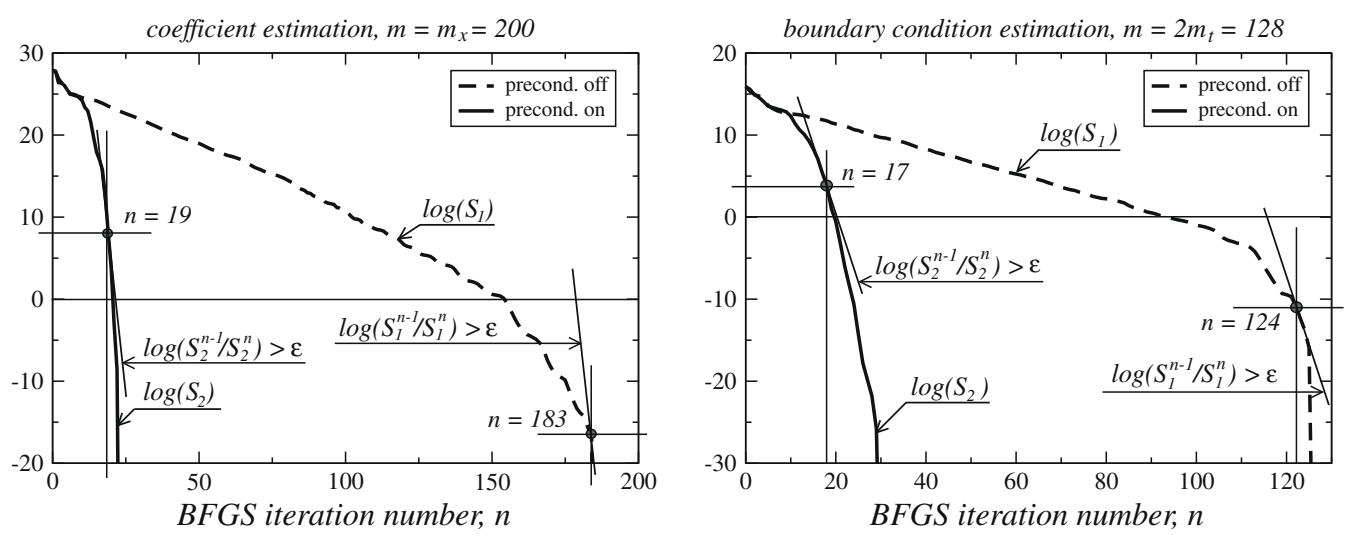

Fig. 15. Convergence history and termination points. Left - diffusion coefficient estimation problem. Right - boundary value control problem.

\subsubsection{Outflow driving boundary}

A distinguishing feature of this case is that the driving boundary at $x=0$ is the outflow boundary (i.e. $w<0(|w|=2)$ ), therefore perturbations at the boundary do not propagate far into the domain. The field variable $\bar{\varphi}(x, t)$ for this case is presented in Fig. 12(left), the diffusion coefficient $k(\bar{\varphi}(x, t))-$ in Fig. 12(right).

We compute the $H$-covariance and the ensemble covariance assuming the following properties of errors $\xi_{i}: \alpha=1.0, \gamma=10.0, \sigma_{o b s}=0.03$. The variances are presented in Fig. 13. Once again, one can notice that the flux at inflow boundary $x=1$ can be well identified, which is a result one should expect. An unexpected result is that the flux $u_{1}$ at outflow boundary $x=0$ can also be partly identified (because of the nonlinearity). Moreover, we know exactly which parts of the boundary (when) and how well this can be done. Therefore, in order to analyse numerically the degree of uncertainty reduction in a model parameter (control), the $\mathrm{H}$-covariance has to be computed. The similar theoretical analysis is often a difficult task, for the nonlinear case in particular.

The $H$-covariance and the corresponding ensemble covariance matrices are presented in Fig. 14, upper panel (for the outflow boundary, $x=0$ ) and lower panel (for the inflow boundary, $x=1$ ). A satisfactory agreement between the $H$-covariance and the ensemble covariance in both cases can be noticed (as well as for the variances presented in Fig. 13).

\subsection{Benefits of preconditioning}

The preconditioning technique is described in Section 5.2. As a preconditioner we use the Cholesky factor of the weight matrix $V_{1}$, which is inverse to the background error covariance matrix $V_{\xi_{1}}$. In [6] we reported that as the correlation radius of the background error (controlled by $\gamma$ ) grows, the number of iterations required to form the inverse Hessian quickly increases approaching the number of unknowns. This is true for the problems considered in this paper if the original auxiliary problem (3.24) and (3.25) or (4.20) and (4.21) is considered. However, if we solve the modified auxiliary DA problem for the preconditioned Hessian (5.5) and (5.6) or (5.8) and (5.9), the quasi-Newton approximation of the inverse Hessian requires much fewer BFGS updates to be formed. Examples of the convergence history with and without preconditioning (for otherwise equivalent conditions) are presented in Fig. 15 for the diffusion coefficient estimation problem (left), and for the boundary flux estimation problem (right). These two cases correspond to the problems considered earlier and presented in Fig. 4(left) and in Fig. 10(right), respectively.

The criteria used to stop the BFGS iterations takes into account the slope of the convergence curve

$$
\log \left(\frac{S_{i}^{n-1}}{S_{i}^{n}}\right)>\epsilon, \quad i=1,2
$$

where $n$ is the BFGS iteration number, $S_{i}^{n}$ is the corresponding value of the cost functional $S_{i}$. The value of the threshold $\epsilon$ used in all examples was $\epsilon=4$. In the vast majority of numerical tests this criterion leads to accurate results.

A major advantage of using the inverse BFGS update formula is that one can compute the $H$-covariance using the product $H v$, that is without the need to form and keep the matrix $H$. However, if the number of the BFGS iterations is large, this advantage would be quickly annihilated because of the need to keep a large number of updates (pair-vectors), and to run both the TLM and adjoint models at each iteration. The examples of the convergence history presented in Fig. 15 show that with an efficient preconditioning the $H$-covariance matrix can be computed in a number of iterations much less than the number of unknowns $m$, therefore much less memory is required to keep the updates. Let us recall that the direct method for computing the covariance via the Hessian matrix $H$ (sometimes referred to as the Fisher information matrix) would require all $m$ runs of the TLM plus the inversion of the matrix $H$. 


\section{Conclusions}

In [6] we investigated the initial-value optimal control problem for a nonlinear evolution model, which is a typical DA problem arising in geophysics. In this paper we consider various parameter estimation problems such as a distributed coefficient estimation problem and a boundary value estimation problem. These two are different types of problem that may arise in the same application field. The first one is often considered to calibrate models which describe the transport of traces or turbulent dissipation processes in ocean and atmosphere, the second one - to calibrate regional scale (coastal, for example) flow models. The optimal solution error a posteriori covariance matrix (approximated by the $H$-covariance) can be used for a subsequent DA, as an optimal solution quality estimate, for the analysis of uncertainty in model outputs (either forecast or hind-cast) and for optimal design of observation schemes, including the adaptive real-time mode. Besides, it can be used to quantify the identifiability levels for any estimation problem, and therefore, it is an important (numerical) analysis tool which may complement existing analytical methods. We found that the $\mathrm{H}$-covariance and the uncertainty reduction quotient $\zeta$ in particular are convenient graphic measures better suited for visual analysis and interpretation than the spectrum of the Hessian, for example. Let us notice that even though the idea of using the a posteriori covariance for numerical analysis of identifiability of large-scale complex nonlinear dynamical systems looks apparent, we are not aware of studies exploiting this idea in practice.

The main theoretical result presented in [6] is that in the nonlinear case the analysis (initial-value) error covariance operator can be approximated by the inverse Hessian of the auxiliary DA problem based on the TLM constraints. This result was first formulated in an operator form in [6]. In the present paper we prove the same result for parameter estimation problems. It can be stated that for any DA problem, whatever the data, controls and constraints are, the same results should be valid (if the TLM of the constraints exists and the Hessian of the auxiliary problem is invertible).

In [6] we presented details of a numerical algorithm to compute an estimate of the covariance as the inverse Hessian with the use of the quasi-Newton BFGS minimization method. We refer to this estimate as the ' $H$-covariance'. In this paper we formulate a modified auxiliary DA problem for a preconditioned Hessian, solve this problem using the BFGS method and recover the inverse Hessian of the original auxiliary DA problem, i.e. the $\mathrm{H}$-covariance matrix. Let us note that one may need to recover only target elements of this matrix. As a preconditioner we use the Cholesky factor of the background error weight matrix $V_{1}$. Numerical experiments show that the preconditioning allows the number of iterations required to build an accurate quasi-Newton approximation of the inverse Hessian to be reduced by an order of magnitude (as compared to the number of unknowns). With the use of efficient preconditioning, the suggested method becomes significantly more attractive from the computational point of view than any existing direct method (i.e. a method which would require the full matrix $H$ to be computed and stored).

We suggest an approach for specifying directly the background error weight matrix $V_{1}$ (inverse of the background error covariance matrix). This method is based on the assumption that the background error belongs to the Sobolev space. It is found that with the use of the weight functions $\alpha(z)$ and $\gamma(z)$ in (5.1) one can generate a family of the covariance matrices, such that $\alpha(z)$ controls mainly the variance and $\gamma(z)$ controls mainly the correlation radius. Therefore, given a background error covariance matrix $V_{\xi_{1}}$ (generally dense), one can generate a corresponding sparse weight matrix $V_{1}$ such that $V_{1}^{-1} \approx V_{\xi_{1}}$. This is important since we may need the (incomplete) Cholesky factors of $V_{1}$ for the preconditioning.

We conducted a series of numerical tests to validate the performance of the BFGS algorithm by comparing the $H$-covariance against the ensemble covariance. Generally, we observed that those two are in a good agreement. This confirms that the linearization error, even though locally significant, does not result in equally significant deviations in the $H$-covariance. In one example we do observe a noticeable difference that vanishes as the magnitude of the input errors is reduced. As before ([6]), the influence of the background error correlation radius on the $H$-covariance is found to be crucial.

The $H$-covariance can be used to investigate numerically the degree of uncertainty reduction and to design efficient observation schemes including adaptive observations. The numerical tests performed have revealed some interesting features of the estimation problems under consideration. For example, one practical conclusion is that in order to identify the diffusion coefficient at a certain location, one needs a pair of closely located field sensors. Another surprising result is a partial identifiability of the outflow boundary in the nonlinear convection-diffusion model, which is a consequence of the nonlinearity. One should expect that for more complex models more interesting properties are likely to be discovered.

\section{Acknowledgements}

The first author was funded through the Glasgow Research Partnership in Engineering (GRPE) by the Scottish Funding Council. The two co-authors were funded by the MOISE project (CNRS, INRIA, UJF, INPG), ADAMS project and the project 09-01-00284 of the Russian Foundation for Basic Research and within the programme ECO-NET (EGIDE). The authors also acknowledge the partial support of the Sir David Anderson Bequest (University of Strathclyde).

\section{References}

[1] O.M. Alifanov, E.A. Artyukhin, S.V. Rumyantsev, Extreme Methods for Solving Ill-posed Problems with Applications to Inverse Heat Transfer Problems, Begel House Publishers, 1996.

[2] G. Chavent, Local stability of the output least square parameter estimation technique, Math. Appl. Comput. 2 (1983) 3-22. 
[3] P. Courtier, J.N. Thepaut, A. Hollingsworth, A strategy for operational implementation of 4D-Var, using an incremental approach, Quart. J. Roy. Meteor. Soc. 120 (1994) 1367-1387.

[4] M. Fisher, P. Courtier, Estimating the covariance matrices of analysis and forecast error in variational data assimilation, ECMWF Res. Dept. Techn. Memo. (1995) 220.

[5] M. Fisher, J. Nocedal, Y. Trémolet, S.J. Wright, Data assimilation in weather forecasting: a case study in PDE-constrained optimization. Optim. Eng., 2008. doi:10.1007/s11081-008-9051-5.

[6] I. Gejadze, F.-X. Le Dimet, V. Shutyaev, On analysis error covariances in variational data assimilation, SIAM J. Sci. Comput. 30 (4) (2008) $1847-1874$.

[7] J.R. Gunson, P. Malanotte-Rizzoli, Assimilation studies of open-ocean flows. 2. Error measures with strongly nonlinear dynamics, J. Geophys. Res. 101 (C12) (1996) 28473-28488.

[8] L. Hascoët, V. Pascual, TAPENADE 2.1 user's guide, INRIA Tech. Rep., 2004, no.0300, 78 pp.

[9] F.-X. Le Dimet, I.M. Navon, D.N. Daescu, Second-order information in data assimilation, Monthly Weather Rev. 130 (3) (2002) $629-648$.

[10] F.X. Le Dimet, O. Talagrand, Variational algorithms for analysis and assimilation of meteorological observations: theoretical aspects, Tellus 38A (1986) $97-110$.

[11] F.-X. Le Dimet, V. Shutyaev, On deterministic error analysis in variational data assimilation, Nonlinear Process. Geophys. 14 (2005) 1-10.

[12] J.L. Lions, Contrôle Optimal des Systèmes Gouvernés par des Équations aux Dérivées Partielles, Dunod, Paris, 1968.

[13] G.I. Marchuk, V.I. Agoshkov, V.P. Shutyaev, Adjoint Equations and Perturbation Algorithms in Nonlinear Problems, CRC Press Inc., New York, 1996.

[14] I.M. Navon, Practical and theoretical aspects of adjoint parameter estimation and identifiability in meteorology and oceanography, Dyn. Atmos. Oceans. Special Issue in Honor of Richard Pfeffer 27 (1998) 55-79.

[15] S.V. Patankar, Numerical Heat Transfer and Fluid Flow, Hemisphere Publishing Corporation, New York, 1980.

[16] E. Polak, Optimization: Algorithms and Consistent Approximations, Applied Mathematical Sciences, vol. 124, Springer, New York, 1997.

[17] F. Rabier, P. Courtier, Four-dimensional assimilation in the presence of baroclinic instability, Quart. J. Roy. Meteorol. Soc. 118 (1992) $649-672$.

[18] A. Sandu, L. Zhang, Discrete second order adjoints in atmospheric chemical transport modeling, J. Computat. Phys. 227 (12) (2008) $5949-5983$.

[19] V.P. Shutyaev, F.-X. Le Dimet, I.Yu. Gejadze, A posteriori error covariances in variational data assimilation, Russ. J. Numer. Anal. Math. Modell. 24 (2) (2009) 161-169.

[20] N-Z. Sun, Inverse Problems in Groundwater Modeling, Kluwer, Dordrecht, 1994.

[21] W.C. Thacker, The role of the Hessian matrix in fitting models to measurements, J. Geophys. Res. 94 (C5) (1989) 6177-6196.

[22] J.N. Thepaut, P. Courtier, Four-dimensional variational assimilation using the adjoint of a multilevel primitive equation model, Quart. J. Roy. Meteorol. Soc. 117 (1991) 1225-1254.

[23] A.N. Tikhonov, Solution of incorrectly formulated problems and the regularization method, Dokl. Akad. Nauk. 151 (1963) $501-504$.

[24] J. Tshimanga, S. Gratton, A.T. Weaver, A. Sartenaer, Limited-memory preconditioners, with application to incremental four-dimensional variational data assimilation, Quart. J. Roy. Meteorol. Soc. 134 (2008) 751-769.

[25] Y. Zhu, I.M. Navon, Impact of parameter estimation on the performance of the FSU global spectral model using its full-physics adjoint, Monthly Weather Rev. 127 (1999) 1497-1517.

[26] Y. Yang, I.M. Navon, P. Courtier, A new Hessian preconditioning method applied to variational data assimilation experiments using NASA general circulation model, Monthly Weather Rev. 124 (1996) 1000-1017. 